\title{
LOCAL AND GLOBAL TAMENESS IN KRULL MONOIDS
}

\author{
WEIDONG GAO, ALFRED GEROLDINGER, AND WOLFGANG A. SCHMID
}

\begin{abstract}
Let $H$ be a Krull monoid with finite class group $G$ such that every class contains a prime divisor. Then the global tame degree $\mathrm{t}(H)$ equals zero if and only if $H$ is factorial (equivalently, $|G|=1$ ). If $|G|>1$, then $\mathrm{D}(G) \leq \mathrm{t}(H) \leq 1+\mathrm{D}(G)(\mathrm{D}(G)-1) / 2$, where $\mathrm{D}(G)$ is the Davenport constant of $G$. We analyze the case when $\mathrm{t}(H)$ equals the lower bound, and we show that $\mathrm{t}(H)$ grows asymptotically as the upper bound, when both terms are considered as functions of the rank of $G$. We provide more precise results if $G$ is either cyclic or an elementary 2-group.
\end{abstract}

\section{Dedicated to Marco Fontana on the occasion of his 65th birthday}

\section{INTRODUCTION}

In an atomic monoid, every non-unit can be written as a finite product of atoms (irreducible elements). The multiplicative monoid of non-zero elements from a noetherian domain is such an atomic monoid, and furthermore it is a Krull monoid if and only if the domain is integrally closed. In a given monoid $H$, all factorizations into atoms are unique (in other words, $H$ is factorial) if and only if $H$ is a Krull monoid with trivial class group. Otherwise, the non-uniqueness of factorizations is described by arithmetical invariants, such as sets of lengths, catenary and tame degrees.

The concepts of local and global tameness have found some attention in recent literature, and they were studied in settings ranging from numerical monoids to noetherian domains (confer [5, 14, 4, 15, 11, 23, 24, 2, 21, 18, 22). We recall their definitions. Let $H$ be a monoid and $u \in H$ an atom. Then the local tame degree $\mathrm{t}(H, u)$ is the smallest $N$ with the following property: for any multiple $a$ of $u$ and any factorization $a=v_{1} \cdot \ldots \cdot v_{n}$ of $a$, which does not contain the $u$, there is a short subproduct which is a multiple of $u$, say $v_{1} \cdot \ldots \cdot v_{m}$, and a refactorization of this subproduct which contains $u$, say $v_{1} \cdot \ldots \cdot v_{m}=u u_{2} \cdot \ldots \cdot u_{\ell}$, such that $\max \{\ell, m\} \leq N$. Thus the local tame degree $\mathrm{t}(H, u)$ measures the distance between any factorization of a multiple $a$ of $u$ and a factorization of $a$ which contains the $u$. The global tame degree $\mathrm{t}(H)$ is the supremum of the local tame degrees over all atoms $u \in H$, and $H$ is called (globally) tame if the global tame degree $\mathrm{t}(H)$ is finite.

Local tameness is a basic finiteness property in the theory of non-unique factorizations in the sense that in many settings where an arithmetical finiteness property has to be derived, local tameness has to be proved first (confer the proof of the Structure Theorem for sets of lengths in [12, Section 4.3]). Krull monoids with finite class group are globally tame. But if the domain fails to be integrally closed, this does not remain true any more, not even for non-principal orders in number fields. Indeed, a non-principal order $\mathfrak{o}$ in an algebraic number field is always locally tame, but it is globally tame if and only if for every prime ideal $\mathfrak{p}$ containing the conductor there is precisely one prime ideal $\overline{\mathfrak{p}}$ in the principal order $\overline{\mathfrak{o}}$ such that $\overline{\mathfrak{p}} \cap \mathfrak{o}=\mathfrak{p}$ (equivalently, if and only if its elasticity is finite). Higher dimensional analogs will be mentioned after Definition 3.1

2010 Mathematics Subject Classification. 20M13, 13A05, 13F05, 11B75, 11B30.

Key words and phrases. Krull monoids, non-unique factorizations, tame degree, zero-sum subsequence.

This work was supported by NSFC, Project Number 10971108, by the Austrian Science Fund FWF, Project Number P21576-N18, by the Austrian-French Amadée Program FR03/2012, and by the ANR Project Caesar, Project Number ANR-12-BS01-0011. 
The focus of the present paper is on Krull monoids $H$ with finite class group $G$ such that every class contains a prime divisor, and for simplicity suppose now that $|G|>2$. There is the straightforward inequality

$$
\mathrm{D}(G) \leq \mathrm{t}(H) \leq 1+\frac{\mathrm{D}(G)(\mathrm{D}(G)-1)}{2}
$$

where $\mathrm{D}(G)$ is the Davenport constant of $G$. We analyze the case when $\mathrm{t}(H)$ equals the lower bound, and we show that $\mathrm{t}(H)$ grows asymptotically as the upper bound, when both terms are considered as functions of the rank of $G$ (Theorem 4.12). This result, which indicates the general behavior of the tame degree, will be complemented by more precise results if $G$ is either cyclic or an elementary 2-group (Theorems 5.1 and 5.2). Arithmetical invariants (such as sets of lengths, sets of distances, the elasticity, the catenary degree, or the monotone catenary degree) of a Krull monoid as above depend only on the class group $G$ but not on the number of prime divisors in the classes, and therefore all investigations can be carried through in the associated monoid of zero-sum sequences instead of doing them in $H$. In general, this is not the case for the tame degree, and we provide the first example revealing this fact (see Theorem 5.1 but also Proposition 3.3.1 and Remark 3.4.1). Moreover, note that the existing computational methods (as first presented in [5]) cannot be applied to obtain this or many other examples given in the present paper (the problem is the large number of variables involved in the systems of linear Diophantine equations to be solved).

\section{Preliminaries}

Let $\mathbb{N}$ denote the set of positive integers, $\mathbb{P} \subset \mathbb{N}$ the set of prime numbers and $\mathbb{N}_{0}=\mathbb{N} \cup\{0\}$. For real numbers $a, b \in \mathbb{R}$, let $[a, b]=\{x \in \mathbb{Z} \mid a \leq x \leq b\}$ an interval of integers. By a monoid, we mean a commutative semigroup with unit element which satisfies the cancellation laws. All our concepts will be formulated in the language of monoids. The monoids we have in mind are multiplicative monoids of nonzero elements of noetherian or Mori domains, monoids of ideals (with suitable multiplication), and additive monoids of certain classes of modules ([17, 12, 1, 8]).

Arithmetic of monoids. Let $H$ be a monoid. We denote by $\mathrm{q}(H)$ a quotient group of $H$ with $H \subset \mathrm{q}(H)$, by $H^{\times}$the group of invertible elements, and by $H_{\text {red }}=\left\{a H^{\times} \mid a \in H\right\}$ the associated reduced monoid. We say that $H$ is reduced if $H^{\times}=\{1\}$. Furthermore, let $\mathcal{A}(H)$ be the set of atoms (irreducible elements) of $H$. For a set $P$, we denote by $\mathcal{F}(P)$ the free (abelian) monoid with basis $P$. Then every $a \in \mathcal{F}(P)=F$ has a unique representation in the form

$$
a=\prod_{p \in P} p^{\mathrm{v}_{p}(a)} \quad \text { with } \quad \mathrm{v}_{p}(a) \in \mathbb{N}_{0} \text { and } \mathrm{v}_{p}(a)=0 \text { for almost all } p \in P .
$$

We call $\operatorname{supp}_{P}(a)=\operatorname{supp}(a)=\left\{p \in P \mid \mathrm{v}_{p}(a)>0\right\} \subset P$ the support of $a$, and $|a|_{F}=|a|=\sum_{p \in P} \mathrm{v}_{p}(a) \in$ $\mathbb{N}_{0}$ the length of $a$. We will often consider submonoids of free abelian monoids, and in all these situations the length $|\cdot|$ refers to the largest free abelian monoid under consideration. The free monoid $\mathbf{Z}(H)=$ $\mathcal{F}\left(\mathcal{A}\left(H_{\text {red }}\right)\right)$ is called the factorization monoid of $H$, and the unique homomorphism

$$
\pi: \mathrm{Z}(H) \rightarrow H_{\text {red }} \quad \text { satisfying } \quad \pi(u)=u \quad \text { for all } \quad u \in \mathcal{A}\left(H_{\text {red }}\right)
$$

is called the factorization homomorphism of $H$. For $a \in H$, the set

$$
\begin{aligned}
& \mathrm{Z}(a)=\pi^{-1}\left(a H^{\times}\right) \subset \mathrm{Z}(H) \text { is the set of factorizations of } a, \text { and } \\
& \mathrm{L}(a)=\{|z| \mid z \in \mathrm{Z}(a)\} \subset \mathbb{N}_{0} \text { is the set of lengths of } a .
\end{aligned}
$$

By definition, we have $\mathrm{Z}(a)=\{1\}$ and $\mathrm{L}(a)=\{0\}$ for all $a \in H^{\times}$. The monoid $H$ is called

- atomic if $\mathrm{Z}(a) \neq \emptyset$ for all $a \in H$;

- factorial if $|\mathrm{Z}(a)|=1$ for all $a \in H$ (equivalently, $H$ is atomic and every atom is a prime). 
Let $z, z^{\prime} \in \mathbf{Z}(H)$. Then we can write

$$
z=u_{1} \cdot \ldots \cdot u_{\ell} v_{1} \cdot \ldots \cdot v_{m} \quad \text { and } \quad z^{\prime}=u_{1} \cdot \ldots \cdot u_{\ell} w_{1} \cdot \ldots \cdot w_{n}
$$

where $\ell, m, n \in \mathbb{N}_{0}, u_{1}, \ldots, u_{\ell}, v_{1}, \ldots, v_{m}, w_{1}, \ldots, w_{n} \in \mathcal{A}\left(H_{\text {red }}\right)$ are such that $\left\{v_{1}, \ldots, v_{m}\right\} \cap\left\{w_{1}, \ldots, w_{n}\right\}=$ $\emptyset$. The distance between $z$ and $z^{\prime}$ is defined by

$$
\mathrm{d}\left(z, z^{\prime}\right)=\max \{m, n\}=\max \left\{\left|z \operatorname{gcd}\left(z, z^{\prime}\right)^{-1}\right|,\left|z^{\prime} \operatorname{gcd}\left(z, z^{\prime}\right)^{-1}\right|\right\} \in \mathbb{N}_{0} .
$$

Krull monoids. A monoid homomorphism $\varphi: H \rightarrow D$ is called

- a divisor homomorphism if $\varphi(a) \mid \varphi(b)$ implies that $a \mid b$ for all $a, b \in H$.

- cofinal if for every $a \in D$ there exists some $u \in H$ such that $a \mid \varphi(u)$.

- a divisor theory (for $H$ ) if $D=\mathcal{F}(P)$ for some set $P, \varphi$ is a divisor homomorphism, and for every $p \in P$ (equivalently for every $p \in \mathcal{F}(P)$ ), there exists a finite subset $\emptyset \neq X \subset H$ satisfying $p=\operatorname{gcd}(\varphi(X))$.

The quotient group $\mathcal{C}(\varphi)=\mathrm{q}(D) / \mathrm{q}(\varphi(H))$ is called the class group of $\varphi$. For $a \in \mathrm{q}(D)$, we denote by $[a]=[a]_{\varphi}=a \mathrm{q}(\varphi(H)) \in \mathrm{q}(D) / \mathrm{q}(\varphi(H))$ the class containing $a$. If $\varphi: H \rightarrow \mathcal{F}(P)$ is a cofinal divisor homomorphism, then

$$
G_{P}=\{[p]=p \mathrm{q}(\varphi(H)) \mid p \in P\} \subset \mathcal{C}(\varphi)
$$

is called the set of classes containing prime divisors. By its very definition, every glass $g \in \mathcal{C}(\varphi)$ is a subset of $\mathrm{q}(D)$ and $P \cap g$ is the set of prime divisors lying in $g$. The monoid $H$ is called a Krull monoid if it satisfies one of the following equivalent properties ([12, Theorem 2.4.8] or [17, Chapter 22]) :

(a) $H$ is $v$-noetherian and completely integrally closed,

(b) $H$ has a divisor theory,

(c) $H$ has a divisor homomorphism into a free monoid.

If $H$ is a Krull monoid, then a divisor theory is essentially unique and the associated class group depends only on $H$ (it is called the class group of $H$ ). An integral domain $R$ is a Krull domain if and only if its multiplicative monoid $R \backslash\{0\}$ is a Krull monoid, and thus Property (a) shows that a noetherian domain is Krull if and only if it is integrally closed.

The main examples of Krull monoids which we have in mind are those stemming from number theory: rings of integers in algebraic number fields, holomorphy rings in algebraic function fields and regular congruence monoids in these domains are Krull monoids with finite class group such that every class contains infinitely many prime divisors ([12, Section 2.11]). If $R$ is an integral separable finitely generated algebra over an infinite field $k$ such that $\operatorname{dim}_{k}(R) \geq 2$, then $R$ is noetherian and every class contains infinitely many prime divisors ([19]). Monoid domains and power series domains that are Krull are discussed in [20, 3]. For the role of Krull monoids in module theory we refer to [6, 17, 1]. Module theory provides natural examples of Krull monoids where $G_{P} \subsetneq G$ but $G_{P}=-G_{P}$ holds true.

Monoids of zero-sum sequences. Let $G$ be an additive abelian group, $G_{0} \subset G$ a subset and $\mathcal{F}\left(G_{0}\right)$ the free monoid with basis $G_{0}$. According to the tradition of Combinatorial Number Theory, the elements of $\mathcal{F}\left(G_{0}\right)$ are called sequences over $G_{0}$. For a sequence

$$
S=g_{1} \cdot \ldots \cdot g_{\ell}=\prod_{g \in G_{0}} g^{v_{g}(S)} \in \mathcal{F}\left(G_{0}\right),
$$

we call

$$
\sigma(S)=\sum_{i=1}^{\ell} g_{i} \text { the sum of } S \text { and } \Sigma(S)=\left\{\sum_{i \in I} g_{i} \mid \emptyset \neq I \subset[1, \ell]\right\} \text { the set of subsums of } S .
$$

Furthermore, $S$ is called zero-sum free if $0 \notin \Sigma(S)$, and it is a minimal zero-sum sequence if $|S| \geq 1$, $\sigma(S)=0$ and $\sum_{i \in I} g_{i} \neq 0$ for all $\emptyset \neq I \subsetneq[1, \ell]$. The monoid

$$
\mathcal{B}\left(G_{0}\right)=\left\{U \in \mathcal{F}\left(G_{0}\right) \mid \sigma(U)=0\right\}
$$


is called the monoid of zero-sum sequences over $G_{0}$. Since the embedding $\mathcal{B}\left(G_{0}\right) \hookrightarrow \mathcal{F}\left(G_{0}\right)$ is a divisor homomorphism, $\mathcal{B}\left(G_{0}\right)$ is a Krull monoid by Property (c). The monoid $\mathcal{B}(G)$ is factorial if and only if $|G| \leq 2$. For every arithmetical invariant $*(H)$ defined for a monoid $H$, it is usual to write $*\left(G_{0}\right)$ instead of $*\left(\mathcal{B}\left(G_{0}\right)\right.$ ) (whenever the meaning is clear from the context). In particular, we set $\mathcal{A}\left(G_{0}\right)=\mathcal{A}\left(\mathcal{B}\left(G_{0}\right)\right)$, $\mathrm{Z}\left(G_{0}\right)=\mathrm{Z}\left(\mathcal{B}\left(G_{0}\right)\right)$, and $\mathrm{t}\left(G_{0}\right)=\mathrm{t}\left(\mathcal{B}\left(G_{0}\right)\right)$. The atoms of $\mathcal{B}\left(G_{0}\right)$ are precisely the minimal zero-sum sequences over $G_{0}$, and

$$
\mathrm{D}\left(G_{0}\right)=\sup \left\{|U| \mid U \in \mathcal{A}\left(G_{0}\right)\right\} \in \mathbb{N} \cup\{\infty\}
$$

is the Davenport constant of $G_{0}$. Suppose that $G$ is finite with $|G|>1$, say

$$
G \cong C_{n_{1}} \oplus \cdots \oplus C_{n_{r}} \cong C_{q_{1}} \oplus \cdots \oplus C_{q_{s}},
$$

where $r, s \in \mathbb{N}, n_{1}, \ldots, n_{r} \in \mathbb{N}, 1<n_{1}|\ldots| n_{r}$, and $q_{1}, \ldots, q_{s}$ are prime powers (not equal to 1 ). Then $r=\mathrm{r}(G)$ is the rank of $G, s=\mathrm{r}^{*}(G)$ is the total rank of $G, \mathrm{~d}(G)=\mathrm{D}(G)-1$ is the maximal length of a zero-sum free sequence over $G$, and we define

$$
\mathrm{d}^{*}(G)=\sum_{i=1}^{\mathrm{r}(G)}\left(n_{i}-1\right), \mathrm{D}^{*}(G)=\mathrm{d}^{*}(G)+1, \quad \text { and } \quad \mathrm{k}^{*}(G)=\sum_{i=1}^{\mathrm{r}^{*}(G)} \frac{q_{i}-1}{q_{i}} .
$$

Furthermore, we set $\mathrm{d}^{*}(\{0\})=\mathrm{k}^{*}(\{0\})=0$. A straightforward example shows that $\mathrm{D}^{*}(G) \leq \mathrm{D}(G)$. Moreover, equality holds for groups of $\operatorname{rank} r(G) \leq 2$, for $p$-groups, and some other types of groups but not in general ([9] [16]). If $t \in \mathbb{N}$ and $\left(e_{1}, \ldots, e_{t}\right) \in G^{t}$, then $\left(e_{1}, \ldots, e_{t}\right)$ is said to be independent if $e_{1}, \ldots, e_{t}$ are all nonzero and if, for every $\left(m_{1}, \ldots, m_{t}\right) \in \mathbb{Z}^{t}$, the equation $\sum_{i=1}^{t} m_{i} e_{i}=0$ implies that $m_{i} e_{i}=0$ for all $i \in[1, t]$. Furthermore, $\left(e_{1}, \ldots, e_{t}\right)$ is said to be a basis of $G$ if it is independent and $G=\left\langle e_{1}, \ldots, e_{t}\right\rangle$.

\section{TAmeness And Transfer Homomorphisms}

In this section we introduce the concepts of tameness and that of transfer homomorphisms. Our main reference is Section 3.2 in 12 . We present the material in a way suitable for our applications in the following sections. Among others we will show that a Krull monoid is locally tame if and only if the associated block monoid is locally tame, a fact which has not been observed so far. Furthermore, we establish a purely combinatorial characterization of the tame degree of a Krull monoid provided that every class contains sufficiently many prime divisors (Proposition 3.5).

Definition 3.1. Let $H$ be an atomic monoid.

1. For $b \in H$, let $\omega(H, b)$ denote the smallest $N \in \mathbb{N}_{0} \cup\{\infty\}$ with the following property:

For all $n \in \mathbb{N}$ and $a_{1}, \ldots, a_{n} \in H$, if $b \mid a_{1} \cdot \ldots \cdot a_{n}$, then there is a subset $\Omega \subset[1, n]$ such that

$$
|\Omega| \leq N \quad \text { and } \quad b \mid \prod_{\nu \in \Omega} a_{\nu} .
$$

2. For $a \in H$ and $x \in \mathrm{Z}(H)$, let $\mathrm{t}(a, x) \in \mathbb{N}_{0} \cup\{\infty\}$ denote the smallest $N \in \mathbb{N}_{0} \cup\{\infty\}$ with the following property:

If $\mathbf{Z}(a) \cap x \mathbf{Z}(H) \neq \emptyset$ and $z \in \mathbf{Z}(a)$, then there exists $z^{\prime} \in \mathbf{Z}(a) \cap x \mathbf{Z}(H)$ such that $\mathrm{d}\left(z, z^{\prime}\right) \leq N$. For subsets $H^{\prime} \subset H$ and $X \subset Z(H)$, we define

$$
\mathrm{t}\left(H^{\prime}, X\right)=\sup \left\{\mathrm{t}(a, x) \mid a \in H^{\prime}, x \in X\right\} \in \mathbb{N}_{0} \cup\{\infty\} .
$$

$H$ is said to be locally tame if $\mathrm{t}(H, u)<\infty$ for all $u \in \mathcal{A}\left(H_{\text {red }}\right)$.

3. We set

and we call

$$
\omega(H)=\sup \{\omega(H, u) \mid u \in \mathcal{A}(H)\} \in \mathbb{N}_{0} \cup\{\infty\},
$$

$$
\mathrm{t}(H)=\mathrm{t}\left(H, \mathcal{A}\left(H_{\text {red }}\right)\right)=\sup \left\{\mathrm{t}(H, u) \mid u \in \mathcal{A}\left(H_{\text {red }}\right)\right\} \in \mathbb{N}_{0} \cup\{\infty\}
$$


the tame degree of $H$. The monoid $H$ is said to be (globally) tame if $\mathrm{t}(H)<\infty$.

To analyze the above terminology, suppose that $H$ is reduced. By definition, an atom $u \in H$ is prime if and only if $\omega(H, u)=1$. Thus $\omega(H)=1$ if and only if $H$ is factorial, and the $\omega(H, u)$ values measure how far away an atom is from being a prime. Let $a \in H$ and $u \in \mathcal{A}(H)$. If $u \nmid a$, then $\mathrm{t}(a, u)=0$. Otherwise, $\mathrm{t}(a, u)$ is the smallest $N$ with the following property: if $z=v_{1} \cdot \ldots \cdot v_{n}$ is any factorization of $a$ into atoms $v_{1}, \ldots, v_{n}$, then there is a subset $\Omega \subset[1, n]$, say $\Omega=[1, m]$, and a factorization $z^{\prime}=u u_{2} \cdot \ldots \cdot u_{\ell} v_{m+1} \cdot \ldots \cdot v_{n}$ of $a$ with atoms $u_{2}, \ldots, u_{\ell}$ such that $\max \{\ell, m\} \leq N$. If $u$ is a prime, then every factorization of $a$ contains $u$; thus we can choose $z^{\prime}=z$ in the above definition, hence $\mathrm{d}\left(z, z^{\prime}\right)=0$ and $\mathrm{t}(H, u)=0$. If $u$ is not a prime, then $\omega(H, u) \leq \mathrm{t}(H, u)$, and throughout this paper we will use the following characterization of $\mathrm{t}(H, u)$; it is the smallest $N \in \mathbb{N}_{0} \cup\{\infty\}$ with the following property:

If $m \in \mathbb{N}$ and $v_{1}, \ldots, v_{m} \in \mathcal{A}(H)$ are such that $u \mid v_{1} \cdot \ldots \cdot v_{m}$, but $u$ divides no proper subproduct of $v_{1} \cdot \ldots \cdot v_{m}$, then there exist $\ell \in \mathbb{N}$ and $u_{2}, \ldots, u_{\ell} \in \mathcal{A}(H)$ such that $v_{1} \cdot \ldots \cdot v_{m}=u u_{2} \cdot \ldots \cdot u_{\ell}$ and $\max \{\ell, m\} \leq N$ (in other words, $\left.\max \left\{1+\min \mathrm{L}\left(u^{-1} v_{1} \cdot \ldots \cdot v_{m}\right), m\right\} \leq N\right)$.

Globally, we have that $H$ is factorial if and only if $\mathrm{t}(H)=0$, and in the non-factorial case we have $\omega(H) \leq \mathrm{t}(H)$. Moreover, it is not difficult to show that $H$ is tame if and only if $\omega(H)<\infty$ ([14]).

If $H$ is $v$-noetherian, then $\omega(H, b)<\infty$ for all $b \in H$, but this need not be true for the $\mathrm{t}(H, u)$ values. In other words, a $v$-noetherian monoid is not necessarily locally tame. Apart from Krull monoids which will be discussed below, main examples of locally tame monoids are C-monoids: if $R$ is a noetherian domain with integral closure $\bar{R}$, non-zero conductor $\mathfrak{f}$, finite residue field $R / \mathfrak{f}$ and finite class group $\mathcal{C}(\bar{R})$, then $R$ is a C-monoid, and there is an explicit characterization when $R$ is globally tame (see [12, Theorem 2.11.9] and [13, 18, 25]).

A central method to investigate arithmetical phenomena in a given class of monoids $H$ (such as noetherian domains) is to construct a simpler auxiliary monoid $B$ and a homomorphism $\theta: H \rightarrow B$ which will be called a transfer homomorphism and which allows to shift arithmetical properties from $B$ to $H$. The machinery of transfer homomorphisms is most highly developed for Krull monoids but not restricted to them. The auxiliary monoids associated to Krull monoids are monoids of zero-sum sequences over their respective class groups. We start with the necessary definitions.

A monoid homomorphism $\theta: H \rightarrow B$ is called a transfer homomorphism if the following holds:

(T 1) $B=\theta(H) B^{\times}$and $\theta^{-1}\left(B^{\times}\right)=H^{\times}$.

(T 2) If $u \in H, b, c \in B$ and $\theta(u)=b c$, then there exist $v, w \in H$ such that $u=v w, \theta(v) \simeq b$ and $\theta(w) \simeq c$.

A transfer homomorphism $\theta: H \rightarrow B$ between atomic monoids allows a unique extension $\bar{\theta}: \mathrm{Z}(H) \rightarrow \mathbf{Z}(B)$ to the factorization monoids satisfying $\bar{\theta}\left(u H^{\times}\right)=\theta(u) B^{\times}$for all $u \in \mathcal{A}(H)$.

For $a \in H$ and $x \in \mathrm{Z}(H)$, we denote by $\mathrm{t}(a, x, \theta)$ the smallest $N \in \mathbb{N}_{0} \cup\{\infty\}$ with the following property:

If $\mathbf{Z}(a) \cap x \mathbf{Z}(H) \neq \emptyset, \quad z \in \mathbf{Z}(a)$ and $\bar{\theta}(z) \in \bar{\theta}(x) \mathbf{Z}(B)$, then there exists some $z^{\prime} \in \mathbf{Z}(a) \cap x \mathbf{Z}(H)$ such that $\bar{\theta}\left(z^{\prime}\right)=\bar{\theta}(z)$ and $\mathrm{d}\left(z, z^{\prime}\right) \leq N$.

Then

$$
\mathrm{t}(H, x, \theta)=\sup \{\mathrm{t}(a, x, \theta) \mid a \in H\} \in \mathbb{N}_{0} \cup\{\infty\}
$$

is called the tame degree in the fibres. We will make substantial use of this concept in Section 5 .

Lemma 3.2. Let $H$ be a reduced Krull monoid, $H \hookrightarrow F=\mathcal{F}(P)$ a cofinal divisor homomorphism, and let $G_{P} \subset G=F / H$ be the set of all classes containing prime divisors. Let $\widetilde{\boldsymbol{\beta}}: F \rightarrow \mathcal{F}\left(G_{P}\right)$ denote the unique homomorphism defined by $\widetilde{\boldsymbol{\beta}}(p)=[p]$ for all $p \in P$. Further, let $u \in \mathcal{A}(H)$ and $U=\widetilde{\boldsymbol{\beta}}(u)$. 
1. The homomorphism $\boldsymbol{\beta}=\widetilde{\boldsymbol{\beta}} \mid H: H \rightarrow \mathcal{B}\left(G_{P}\right)$ is a transfer homomorphism, and hence

$$
\mathrm{t}\left(G_{P}, U\right) \leq \mathrm{t}(H, u) \leq \mathrm{t}\left(G_{P}, U\right)+\mathrm{t}(H, u, \boldsymbol{\beta}) .
$$

2. $\mathrm{t}(H, u, \boldsymbol{\beta}) \leq 1+|u| \leq 1+\mathrm{D}\left(G_{P}\right)$ for all $u \in \mathcal{A}(H)$.

3. Suppose that $G_{P}=-G_{P}$ and that every nontrivial class contains at least two distinct prime divisors. Then $1+|u|=\mathrm{t}(H, u, \boldsymbol{\beta})$ for all $u \in \mathcal{A}(H)$ with $|u| \geq 3$. In particular, if $\mathrm{D}\left(G_{P}\right) \geq 3$, then $\mathrm{D}\left(G_{P}\right)+1=\max \{\mathrm{t}(H, u, \boldsymbol{\beta}) \mid u \in \mathcal{A}(H)\}$.

Proof. See [12, Theorem 3.2.5 and Proposition 3.4.8] for 1., and [10, Proposition 4.2] for 2. and 3.

For any Krull monoid $H$, we denote by $\widetilde{\boldsymbol{\beta}}: F \rightarrow \mathcal{F}\left(G_{P}\right)$, by $\boldsymbol{\beta}: H \rightarrow \mathcal{B}\left(G_{P}\right)$, and by $\overline{\boldsymbol{\beta}}: \mathbf{Z}(H) \rightarrow \mathbf{Z}\left(G_{P}\right)$ the homomorphisms as defined above.

Proposition 3.3. Let $H$ be a reduced Krull monoid, $H \hookrightarrow F=\mathcal{F}(P)$ a cofinal divisor homomorphism, $G=F / H$, and $G_{P} \subset G$ the set of all classes containing prime divisors such that that $\mathrm{D}\left(G_{P}\right) \geq 2$.

1. $H$ is locally tame if and only if $\mathcal{B}\left(G_{P}\right)$ is locally tame. More precisely, we have

$$
\mathrm{t}\left(G_{P}, U\right) \leq \mathrm{t}(H, u) \leq \mathrm{t}\left(G_{P}, U\right)+|U|+1 \quad \text { for every } \quad u \in \mathcal{A}(H)
$$

2. $\mathrm{t}(H, u) \leq \max \left\{\mathrm{t}\left(G_{P}, U\right), \frac{3+(|u|-1)\left(\mathrm{D}\left(G_{P}\right)-1\right)}{2}\right\} \leq 1+\frac{|u|\left(\mathrm{D}\left(G_{P}\right)-1\right)}{2}$ for every $u \in \mathcal{A}(H)$.

3. $\mathrm{t}\left(G_{P}\right) \leq \mathrm{t}(H) \leq \max \left\{\mathrm{t}\left(G_{P}\right), \frac{3+\left(\mathrm{D}\left(G_{P}\right)-1\right)^{2}}{2}\right\} \leq 1+\frac{\mathrm{D}\left(G_{P}\right)\left(\mathrm{D}\left(G_{P}\right)-1\right)}{2}$, and if $\mathrm{t}(H)>\mathrm{D}\left(G_{P}\right)$, then

$$
\mathrm{t}(H) \leq \max \left\{1+\min \mathrm{L}(W)\left|W \in \mathcal{B}\left(G_{P} \backslash\{0\}\right),\right| W \mid \leq \mathrm{D}\left(G_{P}\right)\left(\mathrm{D}\left(G_{P}\right)-1\right)\right\} .
$$

4. Suppose that $G_{P}=-G_{P}$. If $U \in \mathcal{A}\left(G_{P}\right)$ with $|U| \geq 3$, then $\mathrm{t}\left(G_{P}, U\right) \geq|U|$. In particular, if $\mathrm{D}\left(G_{P}\right) \geq 3$, then $\mathrm{t}\left(G_{P}\right) \geq \mathrm{D}\left(G_{P}\right)$.

Proof. 1. The inequalities follow immediately from Items 1. and 2. of Lemma 3.2 and by the very definition the inequalities show that $H$ is locally tame if and only if $\mathcal{B}\left(G_{P}\right)$ is locally tame.

2. See [12, Theorem 3.4.10.6].

3. The first inequalities follow immediately from 1. and 2. Suppose that $\mathrm{t}(H)>\mathrm{D}\left(G_{P}\right)$. If $\mathrm{t}(H)$ is infinite, then $\mathrm{D}\left(G_{P}\right)$ is necessarily also infinite and the last inequality is clearly true. So, suppose $\mathrm{t}(H)$ is finite. There are atoms $u, u_{2}, \ldots u_{\ell}, v_{1}, \ldots, v_{m}$ such that $u \mid v_{1} \ldots v_{m}$, but $u$ divides no proper subproduct, and $\mathrm{t}(H)=\mathrm{t}(H, u)=\max \{\ell, m\}$. Since $m \leq|u| \leq \mathrm{D}\left(G_{P}\right)$, it follows that $\mathrm{t}(H, u)=\ell=1+\min \mathrm{L}(w)$ with $w=u^{-1} v_{1} \cdot \ldots \cdot v_{m}$. Since

$$
|w|=\left|v_{1} \cdot \ldots \cdot v_{m}\right|-|u| \leq|u| \mathrm{D}\left(G_{P}\right)-|u| \leq \mathrm{D}\left(G_{P}\right)\left(\mathrm{D}\left(G_{P}\right)-1\right),
$$

the assertion follows.

4. Suppose that $U=g_{1} \cdot \ldots \cdot g_{m}$ with $m \geq 3$, and set $V_{i}=\left(-g_{i}\right) g_{i}$ for all $i \in[1, m]$. Then $U \mid V_{1} \cdot \ldots \cdot V_{m}$, but $U$ divides no proper subproduct. Since $U(-U)=V_{1} \cdot \ldots \cdot V_{m}$, it follows that $\mathrm{t}\left(G_{P}, U\right) \geq \max \{2, m\}=m=|U|$. The statement on $\mathrm{t}\left(G_{P}\right)$ is an immediate consequence.

Remarks 3.4. 1. Proposition 3.3 shows that the property whether $H$ is locally tame or not depends only on $G_{P}$. This is not true for global tameness. We argue as follows. By [15, Example 4.13], there is a tame Krull monoid $H^{\prime}$ with class group $G^{\prime}$, set of prime divisors $G_{P}^{\prime} \subset G^{\prime}$ such that $D\left(G_{P}^{\prime}\right)=\infty$. Since $H^{\prime}$ is tame, $\mathcal{B}\left(G_{P}^{\prime}\right)$ is tame by Proposition 3.3.3. By a Realization Theorem for Krull monoids ([12, Theorem 2.5.4]), there is a Krull monoid $H$ with class group $G$, set of prime divisors $G_{P} \subset G$, and an isomorphism $\Phi: G \rightarrow G^{\prime}$ with $\Phi\left(G_{P}\right)=G_{P}^{\prime}$ such that every class in $G_{P}$ contains at least two distinct prime divisors. Then [15, Theorem 4.2] implies that $H$ is not tame, but $\mathcal{B}\left(G_{P}\right) \cong \mathcal{B}\left(G_{P}^{\prime}\right)$ is tame.

2. Statement 3 of Proposition 3.3 shows that the finiteness of the Davenport constant implies that $H$ is globally tame and hence locally tame. Note, if $G_{P}$ is finite, then $\mathrm{D}\left(G_{P}\right)$ is finite, and the converse 
holds if $G$ has finite total rank ([12, Theorem 3.4.2]). Moreover, if $G_{P}=G$, then $G$ is finite if and only if $\mathrm{D}(G)$ is finite if and only if $H$ is locally tame if and only if $H$ is tame ([14, Theorem 4.4]).

As mentioned above, we can have that $\mathrm{t}\left(G_{P}\right)<\infty=\mathrm{t}(H)$. We will also give an example of a finite abelian class group such that $\mathrm{t}(G)<\mathrm{t}(H)$ (see Theorem5.1). Thus in general the tame degree $\mathrm{t}(H)$ does not coincide with the tame degree of the associated monoid of zero-sum sequences. However, if every class contains sufficiently many prime divisors, then the following proposition offers a characterization of $\mathrm{t}(H)$ in terms of zero-sum theory. This opens the door to study the arithmetical invariant $\mathrm{t}(H)$ with methods from Combinatorial and Additive Number Theory.

Proposition 3.5. Let $H$ be a Krull monoid with class group $G$ and let $G_{P} \subset G$ denote the set of classes containing prime divisors. Suppose that $G_{P}=-G_{P}$ and that $2<\mathrm{D}\left(G_{P}\right)<\infty$.

1. Let $u \in \mathcal{A}(H)$ and $U=\boldsymbol{\beta}(u)$ with $|U| \geq 3$. If every nontrivial class contains at least $|U|+1$ distinct prime divisors, then

$$
\begin{gathered}
\mathrm{t}(H, u)=\max \left\{|U|, 1+\min \mathrm{L}\left(A_{1} \cdot \ldots \cdot A_{m}\right) \mid m \in \mathbb{N}, U=S_{1} \cdot \ldots \cdot S_{m} \text { and, for all } i \in[1, m],\right. \\
\left.S_{i}, A_{i} \in \mathcal{F}\left(G_{P}\right) \backslash\{1\} \text { with } S_{i} A_{i} \in \mathcal{A}\left(G_{P}\right)\right\} .
\end{gathered}
$$

2. If every nontrivial class contains at least $\mathrm{D}\left(G_{P}\right)+1$ distinct prime divisors, then

$$
\begin{aligned}
& \mathrm{t}(H)=\max \left\{\mathrm{D}\left(G_{P}\right), 1+\right. \min \mathrm{L}\left(A_{1} \cdot \ldots \cdot A_{m}\right) \mid m \in \mathbb{N}, A_{1}, \ldots, A_{m} \in \mathcal{F}\left(G_{P}\right) \backslash\{1\} \\
&\text { are zero-sum free such that } \left.\sigma\left(A_{1}\right) \cdot \ldots \cdot \sigma\left(A_{m}\right) \in \mathcal{A}\left(G_{P}\right)\right\} .
\end{aligned}
$$

Proof. We may suppose that $H$ is reduced, and we consider a divisor theory $H \hookrightarrow F=\mathcal{F}(P)$.

1. First note that

$$
\sigma\left(A_{1} \cdot \ldots \cdot A_{m}\right)=\sigma\left(A_{1}\right)+\ldots+\sigma\left(A_{m}\right)=-\sigma(U)=0,
$$

hence $A_{1} \cdot \ldots \cdot A_{m} \in \mathcal{B}\left(G_{P}\right)$. Since $S_{i} A_{i} \in \mathcal{A}\left(G_{P}\right)$ and $S_{i} \neq 1$, it follows that $\left|A_{i}\right| \leq \mathrm{D}\left(G_{P}\right)-1$ for all $i \in[1, m]$, and hence $\left|A_{1} \cdot \ldots \cdot A_{m}\right| \leq m\left(\mathrm{D}\left(G_{P}\right)-1\right) \leq|U|\left(\mathrm{D}\left(G_{P}\right)-1\right)$. Thus we get

$$
\min \mathrm{L}\left(A_{1} \cdot \ldots \cdot A_{m}\right) \leq\left|A_{1} \cdot \ldots \cdot A_{m}\right| / 2 \leq\left(\mathrm{D}\left(G_{P}\right)-1\right) \mathrm{D}\left(G_{P}\right) / 2 .
$$

Thus the set $\{|U|, 1+\min \mathrm{L}(\cdot) \mid \ldots\}$ is finite, and we denote by $t^{\prime}$ its maximum.

First we show that $\mathrm{t}(H, u) \leq t^{\prime}$. Let $a \in H$ with $\mathrm{t}(H, u)=\mathrm{t}(a, u)$. Let $\ell, m \in \mathbb{N}, v_{1}, \ldots, v_{m}, u_{2}, \ldots, u_{\ell} \in$ $\mathcal{A}(H)$ such that $u \mid v_{1} \cdot \ldots \cdot v_{m}$, but $u$ divides no proper subproduct, $v_{1} \cdot \ldots \cdot v_{m}=u u_{2} \cdot \ldots \cdot u_{\ell}$ and $\max \{\ell, m\}=\mathrm{t}(a, u)$. If $\ell \leq m$, then $\mathrm{t}(a, u)=m \leq|U| \leq t^{\prime}$. Suppose that $\ell>m$. Then $\mathrm{t}(a, u)=\ell$ and $\ell-1=\min \mathrm{L}\left(u^{-1} a\right)$. Since $u$ divides $v_{1} \ldots v_{m}$ but no proper subproduct, there are $s_{1}, \ldots, s_{m}, a_{1}, \ldots, a_{m} \in$ $F \backslash\{1\}$ such that $u=s_{1} \cdot \ldots \cdot s_{m}$, and $v_{i}=s_{i} a_{i}$ for all $i \in[1, m]$. Setting $S_{i}=\boldsymbol{\beta}\left(s_{i}\right)$ and $A_{i}=\boldsymbol{\beta}\left(a_{i}\right)$ for all $i \in[1, m]$ we obtain that

$$
\mathrm{t}(H, u)=\ell=1+\min \mathrm{L}\left(u^{-1} a\right)=1+\min \mathrm{L}\left(\boldsymbol{\beta}\left(u^{-1} a\right)\right)=1+\min \mathrm{L}\left(A_{1} \cdot \ldots \cdot A_{m}\right) \leq t^{\prime} .
$$

Next we show that $t^{\prime} \leq \mathrm{t}(H, u)$. If $t^{\prime}=|U|$, then the statement follows from Proposition 3.3. Suppose that $t^{\prime}>|U|$, and let $S_{1}, A_{1}, \ldots, S_{m}, A_{m}$ be as in the definition of $t^{\prime}$ such that $t^{\prime}=1+\min \mathrm{L}\left(A_{1} \cdot \ldots\right.$. $\left.A_{m}\right)$. There are $s_{1}, \ldots, s_{m} \in F \backslash\{1\}$ such that $u=s_{1} \cdot \ldots \cdot s_{m}$ and $S_{i}=\boldsymbol{\beta}\left(s_{i}\right)$ for all $i \in[1, m]$. Set $\gamma=\left|A_{1} \cdot \ldots \cdot A_{m}\right|$. Since every class contains at least $|U|+1$ distinct prime divisors, there are primes $p_{1}, \ldots, p_{\gamma} \in P \backslash \operatorname{supp}_{P}(u)$ and elements $a_{1}, \ldots, a_{m} \in F$ such that $a_{1} \cdot \ldots \cdot a_{m}=p_{1} \cdot \ldots \cdot p_{\gamma}$, $\operatorname{gcd}_{F}\left(u, a_{1} \cdot \ldots \cdot a_{m}\right)=1$, and $\boldsymbol{\beta}\left(a_{i}\right)=A_{i}$ for all $i \in[1, m]$. Now we define $v_{i}=s_{i} a_{i}$ for all $i \in[1, m]$, and observe that $v_{1}, \ldots, v_{m} \in \mathcal{A}(H)$. By construction, $u \mid v_{1} \cdot \ldots \cdot v_{m}$, but $u$ does not divide any proper subproduct. Let $u_{2}, \ldots, u_{\ell} \in \mathcal{A}(H)$ such that $\max \{\ell, m\} \leq \mathrm{t}(a, u)$. Then

$$
\ell \geq 1+\min \mathrm{L}\left(u^{-1} a\right)=1+\min \mathrm{L}\left(A_{1} \cdot \ldots \cdot A_{m}\right)=t^{\prime}>|U| \geq m,
$$

and hence

$$
\mathrm{t}(H, u) \geq \mathrm{t}(a, u)=\max \{\ell, m\}=\ell \geq t^{\prime} .
$$


2. Let $t^{\prime}$ denote the maximum on the right hand side. First we show that for all $u \in \mathcal{A}(H)$, we have $\mathrm{t}(H, u) \leq t^{\prime}$. We choose $u \in \mathcal{A}(H)$ and use the equation for $\mathrm{t}(H, u)$ derived in 1 . Clearly, we have $|u| \leq \mathrm{D}\left(G_{P}\right) \leq t^{\prime}$. Let $S_{1}, A_{1}, \ldots, S_{m}, A_{m}$ be as in 1 . Since $U=S_{1} \cdot \ldots \cdot S_{m} \in \mathcal{A}\left(G_{P}\right)$, it follows that $U^{\prime}=\sigma\left(S_{1}\right) \cdot \ldots \cdot \sigma\left(S_{m}\right) \in \mathcal{A}\left(G_{P}\right)$ and hence $-U^{\prime}=\sigma\left(A_{1}\right) \cdot \ldots \cdot \sigma\left(A_{m}\right) \in \mathcal{A}\left(G_{P}\right)$. Thus all the assumptions on $A_{1}, \ldots, A_{m}$ of 2 . are satisfied, and thus $\mathrm{t}(H, u) \leq t^{\prime}$.

Conversely, we show that $t^{\prime} \leq \mathrm{t}(H)$. If $t^{\prime}=\mathrm{D}\left(G_{P}\right)$, then $t^{\prime}=\mathrm{D}\left(G_{P}\right) \leq \mathrm{t}(H, u)$ for some $u \in \mathcal{A}(H)$ with $|u|=\mathrm{D}\left(G_{P}\right)$. Suppose that $t^{\prime}=1+\min \mathrm{L}\left(A_{1} \cdot \ldots \cdot A_{m}\right)$ with $A_{1}, \ldots, A_{m}$ be as in 2 . For $i \in[1, m]$, we define $S_{i}=-\sigma\left(A_{i}\right)$, and we set $U=S_{1} \cdot \ldots \cdot S_{m}$. Then $U, S_{1} A_{1}, \ldots, S_{m} A_{m} \in \mathcal{A}\left(G_{P}\right)$, and for any $u \in \boldsymbol{\beta}^{-1}(U)$, we have $t^{\prime}=1+\min \mathrm{L}\left(A_{1} \cdot \ldots \cdot A_{m}\right) \leq \mathrm{t}(H, u)$ by 1 .

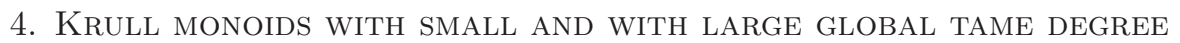

Let $H$ be a Krull monoid with class group $G$ such that every class contains a prime divisor, and suppose that $|G| \geq 3$. Then, by Proposition 3.3 we have

$$
\mathrm{D}^{*}(G) \leq \mathrm{D}(G) \leq \mathrm{t}(G) \leq \mathrm{t}(H) \leq 1+\frac{\mathrm{D}(G)(\mathrm{D}(G)-1)}{2} .
$$

The main result in this section is Theorem 4.12. It characterizes when the equality $\mathrm{D}^{*}(G)=\mathrm{t}(G)$ and when the equality $\mathrm{D}^{*}(G)=\mathrm{t}(H)$ do hold. These characterizations reveal the first example showing that $\mathrm{t}(G)<\mathrm{t}(H)$ may happen. On the other hand, if we consider $\mathrm{t}(H)$ and $\mathrm{D}(G)$ as functions of the rank $r$ of $G$ (with fixed exponent), then $\mathrm{t}(H)$ is growing as the upper bound given above.

We start with two lemmas providing lower bounds for the global tame degree.

Lemma 4.1. Let $G$ be a finite abelian group with $|G|>1$.

1. Then $\mathrm{t}(G) \geq 1+\exp (G) \mathrm{k}^{*}(G)$.

2. If $G$ is cyclic of order $|G|=n \geq 25$, then $\mathrm{t}(G)>2 n-7 \sqrt{n}+10$.

Proof. 1. See [12, Proposition 6.5.2].

2. Let $G$ be cyclic of order $|G|=n \geq 25$. We start with a special construction (which is very similar to [12, Proposition 6.5.2]). We set $n=q m+j$, where $q \in[2, n-2]$ with $\operatorname{gcd}(q, n)=1$, and $m, j \in \mathbb{N}$, and choose a non-zero element $g \in G$. The atoms

$$
U=(q g)^{n}, U_{0}=g^{n}, U_{1}=(-g) g, V=(q g) g^{n-q}, V^{\prime}=(q g)(-g)^{q}
$$

are pairwise distinct, and we have

$$
A=V^{n-m} V^{\prime m}=U U_{1}^{q m} U_{0}^{n-q-m} \in \mathcal{B}(G) .
$$

Since $\left|\mathrm{Z}\left(U_{1}^{q m} U_{0}^{n-q-m}\right)\right|=1, A$ has precisely one factorization which is divisible by $U$. Therefore we obtain that

$$
\begin{aligned}
\mathrm{t}(G) & \geq \mathrm{t}(A, U) \geq \mathrm{d}\left(V^{n-m} V^{\prime m}, U U_{1}^{q m} U_{0}^{n-q-m}\right) \\
& =\max \{n, 1+q m+n-q-m\}=n+(q-1)(m-1) .
\end{aligned}
$$

Thus, it would remain to find $q, m$ and $j$ fulfilling the relevant conditions such that $n+(q-1)(m-1)$ is greater than $2 n-7 \sqrt{n}+10$. The main obstacle here is that $q$ needs to be co-prime to $n$. To side-step this problem for the most part, we first apply the first part of this result.

It is easy to see that if $n$ is divisible by at least three distinct primes then $\mathrm{k}(G) \geq 2-1 / n$, and the result follows directly from the first part.

So, we may assume that $n$ is divisible by at most two distinct primes. For such an $n$ it is well-known and not hard to see that among each four consecutive integers there is at least one co-prime to $n$. Indeed, consider two distinct primes $p, q$, and assume for a contradiction $a, a+1, a+2, a+3$ are all divisible by $p$ or $q$, say $p \mid a$, then $p \nmid(a+1)$ so $q \mid a+1$ implying that $p$ needs to divide $a+2$ and thus $p=2$ and $q \neq 2$, and $a+3$ is divisible neither by $p$ nor by $q$, a contradiction. 
Thus, we can choose some $q$ that is co-prime to $n$ from the set $\{\lfloor\sqrt{n}\rfloor-3, \ldots,\lfloor\sqrt{n}\rfloor\}$; note that here we use the condition $n \geq 25$ to ensure that these elements are at least 2 .

We then set $m=\lfloor n / q\rfloor$, the quotient of the Euclidean division of $n$ by $q$, and $j$ the rest (note that $j$ is non-zero as $q$ does not divide $n)$. It follows that $m \geq\lfloor\sqrt{n}\rfloor$.

From this we get that $(m-1)(q-1)>(\sqrt{n}-2)(\sqrt{n}-5)$, and the claim follows.

It is apparent from the proof that also for $n<25$ non-trivial bounds can be obtained using the same method. However, if one wishes to have a bound for some specific (small) values of $n$, one should in any case rather use the bound $n+(q-1)(m-1)$ directly for an in this case suitable choice of $q$, or at least not use the crude estimate $\lfloor\sqrt{n}\rfloor>\sqrt{n}-1$ so that we made no effort to avoid the condition $n \geq 25$. Moreover, for $n$ a prime or also a prime-power one can get somewhat better bounds in essentially the same way, using $q=\lfloor\sqrt{n}\rfloor$ or $q \in\{\lfloor\sqrt{n}\rfloor-1,\lfloor\sqrt{n}\rfloor\}$, respectively.

\section{Remark 4.2.}

1. If $G$ is cyclic of order $|G|=n \geq 5$ and $n$ is a prime number, then $\mathrm{t}(G) \geq 2 n-4 \sqrt{n}+4$.

2. If $G$ is cyclic of order $|G|=n \geq 9$ and $n$ is a prime-power, then $\mathrm{t}(G) \geq 2 n-5 \sqrt{n}+6$.

Lemma 4.3. Let $G$ be a finite abelian group, and let $r \in \mathbb{N}$ be even.

1. Let $\left(e_{1}, \ldots, e_{r}\right) \in G^{r}$ be independent such that $\operatorname{gcd}\left(\operatorname{ord}\left(e_{i}\right), \operatorname{ord}\left(e_{j}\right)\right)>1$ for all $i, j \in[1, r]$, $e_{0}=-e_{1}-\ldots-e_{r}, \bar{e}=\sum_{i=1}^{r}(-1)^{i+1} e_{i}$,

$$
U=\bar{e} \prod_{i \in[1, r] \text { odd }}\left(-e_{0}-e_{i}\right) \prod_{i \in[1, r] \text { even }}\left(e_{0}+e_{i}\right),
$$

and

$$
\frac{r}{2}=\operatorname{ord}\left(e_{i}\right) k_{i}+j_{i} \quad \text { with } \quad j_{i} \in\left[0, \operatorname{ord}\left(e_{i}\right)-1\right] \quad \text { and } \quad k_{i}=\left\lfloor\frac{r}{2 \operatorname{ord}\left(e_{i}\right)}\right\rfloor \quad \text { for all } i \in[1, r] \text {. }
$$

Then, $U$ is a minimal zero-sum sequence and

$$
\mathrm{t}(G, U) \geq 1+\sum_{i=1}^{r}\left(2\left\lfloor\frac{r}{2 \operatorname{ord}\left(e_{i}\right)}\right\rfloor+j_{i}\right) .
$$

2. If $G=C_{n}^{r}$ with $n \geq 2$ and $\operatorname{gcd}(r-1, n)=1$, then $\mathrm{t}(G) \geq 1+2 r\left\lfloor\frac{r}{2 n}\right\rfloor$ and $\mathrm{t}\left(C_{2}^{r}\right) \geq 1+\frac{r^{2}}{2}$.

Proof. 1. We set $S=\bar{e}^{-1} U$. Since $\sigma(S)=-\bar{e}$, it follows that $U=\bar{e} S$ is a zero-sum sequence. To show that $U$ is a minimal zero-sum sequence it remains to verify that $S$ is zero-sumfree. Assume to the contrary that $S$ has a non-empty zero-sum subsequence

$$
T=\prod_{i \in I_{o}}\left(-e_{0}-e_{i}\right) \prod_{i \in I_{e}}\left(e_{0}+e_{i}\right), \quad \text { where } I_{o} \text { and } I_{e} \text { are subsets of }[1, r] \text { of odd and even numbers, resp. }
$$

For $i \in[1, r]$ let $a_{i} \in\left[0, \operatorname{ord}\left(e_{i}\right)-1\right]$ such that $\sigma(T)=\sum_{i=1}^{r} a_{i} e_{i}$. Recalling the definition of $e_{0}$ we infer that

$$
a_{j} e_{j}=\left(\left|I_{o}\right|-\left|I_{e}\right|+\delta_{j}\right) e_{j} \quad \text { where } \quad \delta_{j}= \begin{cases}-1 & \text { for } j \in I_{o}, \\ 1 & \text { for } j \in I_{e} \\ 0 & \text { otherwise }\end{cases}
$$

Since $\sigma(T)=0$ and $\left(e_{1}, \ldots, e_{r}\right)$ is independent, it follows that $a_{j} e_{j}=0$ for each $j \in[1, r]$, that is $\operatorname{ord}\left(e_{j}\right) \mid\left(\left|I_{o}\right|-\left|I_{e}\right|+\delta_{j}\right)$.

Now, since $T$ is non-empty not all $\delta_{j}$ equal 0 . However, this implies that no $\delta_{j}$ equals 0 . Indeed, if $\delta_{k}=0$ for some $k$, then ord $\left(e_{k}\right)|| I_{o}|-| I_{e} \mid$, and considering some $k^{\prime}$ such that $\delta_{k^{\prime}} \neq 0$ we infer that ord $\left(e_{k^{\prime}}\right)$ divides $\left|I_{o}\right|-\left|I_{e}\right|+1$ or $\left|I_{o}\right|-\left|I_{e}\right|-1$. This entails that $\operatorname{gcd}\left(\operatorname{ord}\left(e_{k}\right), \operatorname{ord}\left(e_{k^{\prime}}\right)\right)$ divides two consecutive integers, a contradiction. Consequently, $I_{o} \cup I_{e}=[1, r], T=S$, and thus $\sigma(T)=\sigma(S)=\bar{e} \neq 0$, a contradiction. 
We set $V_{0}=\bar{e} \prod_{\nu=1}^{r}(-1)^{\nu} e_{\nu}$, and we define

$$
V_{i}= \begin{cases}\left(-e_{0}-e_{i}\right) \prod_{\nu \in[1, r] \backslash\{i\}}\left(-e_{\nu}\right), & \text { if } i \text { is odd, } \\ \left(e_{0}+e_{i}\right) \prod_{\nu \in[1, r] \backslash\{i\}} e_{\nu}, & \text { if } i \text { is even. }\end{cases}
$$

By construction, we have $U \mid \prod_{\nu=0}^{r} V_{\nu}$, but $U$ does not divide any proper subproduct. Furthermore, we have

$$
W=U^{-1} \prod_{\nu=0}^{r} V_{\nu}=\prod_{\nu=1}^{r}\left(\left(-e_{\nu}\right) e_{\nu}\right)^{r / 2} .
$$

Next we study $\mathrm{L}(W)$. For any nonzero $g \in G, k \in \mathbb{N}_{0}$, and $j \in[0, \operatorname{ord}(g)-1]$, we have

$$
\mathrm{L}\left(((-g) g)^{k \operatorname{ord}(g)+j}\right)=\{2 k+\nu(\operatorname{ord}(g)-2)+j \mid \nu \in[0, k]\},
$$

and the minimum of this set equals $2 k+j$. Thus, for every $i \in[1, r]$, we obtain that

$$
\mathrm{L}\left(\left(\left(-e_{i}\right) e_{i}\right)^{r / 2}\right)=\left\{2 k_{i}+\nu\left(\operatorname{ord}\left(e_{i}\right)-2\right)+j_{i} \mid \nu \in\left[0, k_{i}\right]\right\},
$$

with

Since

$$
\min \mathrm{L}\left(\left(\left(-e_{i}\right) e_{i}\right)^{r / 2}\right)=2\left\lfloor\frac{r}{2 \operatorname{ord}\left(e_{i}\right)}\right\rfloor+j_{i}
$$

$$
\mathrm{L}(W)=\sum_{i=1}^{r} \mathrm{~L}\left(\left(\left(-e_{i}\right) e_{i}\right)^{r / 2}\right),
$$

$\min \mathrm{L}(W)$ is the sum of the minima, and it follows that

$$
\mathrm{t}(G, U) \geq 1+\min \mathrm{L}(W)=1+\sum_{i=1}^{r}\left(2\left\lfloor\frac{r}{2 \operatorname{ord}\left(e_{i}\right)}\right\rfloor+j_{i}\right) .
$$

2. Suppose that $G=C_{n}^{r}$ with $r$ and $n$ as above. After choosing a basis $\left(e_{1}, \ldots, e_{r}\right)$ of $G$ with $\operatorname{ord}\left(e_{1}\right)=\ldots=\operatorname{ord}\left(e_{r}\right)=n$, the first inequality follows immediately from 1 . Now suppose that $n=2$. If $r \equiv 0 \bmod 4$, then the statement on $\mathrm{t}\left(C_{2}^{r}\right)$ follows from the first statement. If $r=4 k+2$ with $k \in \mathbb{N}_{0}$, then $r / 2=2 k+1$, and 1 . implies that

$$
\mathrm{t}(G) \geq 1+r(2 k+1)=1+\frac{r^{2}}{2}
$$

Lemma 4.4. Let $G$ be a finite abelian group. Then $\exp (G) \mathrm{k}^{*}(G) \geq \mathrm{d}^{*}(G)$, and equality holds if and only if $G$ is a p-group of the form $G=C_{n}^{r}$ where $n, r \in \mathbb{N}$.

Proof. By definition, the statement holds if $|G|=1$. Suppose that $|G|>1$, say

$$
G \cong C_{n_{1}} \oplus \cdots \oplus C_{n_{r}} \cong C_{q_{1}} \oplus \cdots \oplus C_{q_{s}},
$$

where $r, s \in \mathbb{N}, n_{1}, \ldots, n_{r} \in \mathbb{N}, 1<n_{1}|\ldots| n_{r}$, and $q_{1}, \ldots, q_{s}$ are prime powers. Note that $\exp (G)=$ $n_{r}=\operatorname{lcm}\left(q_{1}, \ldots, q_{s}\right)$. Obviously, the statement holds for cyclic groups of prime power order. Suppose that $G$ is cyclic but not a $p$-group. Then $r=1$ and $s \geq 2$. Since $\frac{q_{j}-1}{q_{j}} \geq \frac{1}{2}$ for all $j \in[1, s]$, it follows that

$$
\sum_{j=1}^{s} \frac{q_{j}-1}{q_{j}} \geq 1 \text { and hence } n_{r} \sum_{j=1}^{s} \frac{q_{j}-1}{q_{j}} \geq n_{r}>\sum_{i=1}^{r}\left(n_{i}-1\right) .
$$

Thus the statement holds for cyclic groups. If $G$ is not cyclic, then

$$
\exp (G) \mathrm{k}^{*}(G)=\exp (G) \sum_{i=1}^{r} \mathrm{k}^{*}\left(C_{n_{i}}\right) \stackrel{(1)}{\geq} \sum_{i=1}^{r} n_{i} \mathrm{k}^{*}\left(C_{n_{i}}\right) \stackrel{(2)}{\geq} \sum_{i=1}^{r} \mathrm{~d}^{*}\left(C_{n_{i}}\right)=\mathrm{d}^{*}(G),
$$


where equality in (1) holds if and only if $n_{1}=\ldots=n_{r}$ and equality in (2) holds if and only if $n_{1}, \ldots, n_{r}$ are prime powers.

Proposition 4.5. Let $G_{1}, G_{2}$ be finite abelian groups with $\mathrm{t}\left(G_{1}\right)>\mathrm{D}\left(G_{1}\right)$.

1. If $\mathrm{t}\left(G_{2}\right)>\mathrm{D}\left(G_{2}\right)$ or $\mathrm{D}\left(G_{2}\right)=\mathrm{D}^{*}\left(G_{2}\right)$, then $\mathrm{t}\left(G_{1} \oplus G_{2}\right) \geq \mathrm{t}\left(G_{1}\right)+\mathrm{t}\left(G_{2}\right)-1$.

2. If $\mathrm{d}\left(G_{1} \oplus G_{2}\right)=\mathrm{d}^{*}\left(G_{1}\right)+\mathrm{d}^{*}\left(G_{2}\right)$, then $\mathrm{t}\left(G_{1} \oplus G_{2}\right)>\mathrm{D}\left(G_{1} \oplus G_{2}\right)$.

Proof. 1. Let $i \in[1,2]$. By definition of $\mathrm{t}\left(G_{i}\right)$, there exists an $U^{(i)} \in \mathcal{A}\left(G_{i}\right)$ with $\mathrm{t}\left(G_{i}\right)=\mathrm{t}\left(G_{i}, U^{(i)}\right)$. By definition of $\mathrm{t}\left(G_{i}, U^{(i)}\right)$, there are $\ell_{i}, m_{i} \in \mathbb{N}, U_{2}^{(i)}, \ldots, U_{\ell_{i}}^{(i)}, V_{1}^{(i)}, \ldots V_{m_{i}}^{(i)} \in \mathcal{A}\left(G_{i}\right)$ such that $U^{(i)} \mid V_{1}^{(i)}$. $\ldots \cdot V_{m_{i}}^{(i)}$, but $U^{(i)}$ divides no proper subproduct, $U^{(i)} U_{2}^{(i)} \cdot \ldots \cdot U_{\ell_{i}}^{(i)}=V_{1}^{(i)} \cdot \ldots \cdot V_{m_{i}}^{(i)}$, and $\mathrm{t}\left(G_{i}, U^{(i)}\right)=$ $\max \left\{\ell_{i}, m_{i}\right\}$. If $\mathrm{t}\left(G_{i}, U^{(i)}\right)=\mathrm{t}\left(G_{i}\right)>\mathrm{D}\left(G_{i}\right)$, then $m_{i} \leq\left|U^{(i)}\right| \leq \mathrm{D}\left(G_{i}\right)$ implies that $m_{i}<\ell_{i}=\mathrm{t}\left(G_{i}, U^{(i)}\right)$ and

$$
\ell_{i}-1=\min \mathrm{L}\left(U_{2}^{(i)} \cdot \ldots \cdot U_{\ell_{i}}^{(i)}\right) .
$$

Now suppose that $\mathrm{t}\left(G_{2}\right)=\mathrm{D}\left(G_{2}\right)=\mathrm{D}^{*}\left(G_{2}\right)$. Then we provide a new construction of the above type where we have $\ell_{2} \geq m_{2}$ and $\ell_{2}-1=\min \mathrm{L}\left(U_{2}^{(2)} \cdot \ldots \cdot U_{\ell_{2}}^{(2)}\right)$. For simplicity we use the same notation as above. Let $\left(e_{1}, \ldots, e_{r}\right)$ be a basis of $G$ such that ord $\left(e_{i}\right)=n_{i}$ for all $i \in[1, r], 1<n_{1}|\ldots| n_{r}$ and $\mathrm{d}^{*}(G)=\sum_{i=1}^{r}\left(n_{i}-1\right)$. We set $e_{0}=e_{1}+\ldots+e_{r}$,

$$
U^{(2)}=\left(-e_{0}\right) e_{0}, V_{1}^{(2)}=e_{0} \prod_{i=1}^{r} e_{i}^{n_{i}-1}, \quad \text { and } \quad V_{2}^{(2)}=-V_{1}^{(2)} .
$$

Then we get $U^{(2)} \prod_{i=1}^{r}\left(\left(-e_{i}\right) e_{i}\right)^{n_{i}-1}=V_{1}^{(2)} V_{2}^{(2)}, \mathrm{t}\left(G_{2}, U^{(2)}\right)=\mathrm{t}\left(G_{2}\right)=1+\mathrm{d}^{*}(G), \ell_{2}=\mathrm{d}^{*}(G)+1 \geq 2=$ $m_{2}$ and

$$
\ell_{2}-1=\min \mathrm{L}\left(U_{2}^{(2)} \cdot \ldots \cdot U_{\ell_{2}}^{(2)}\right)
$$

with the obvious definition of $U_{2}^{(2)}, \ldots, U_{\ell_{2}}^{(2)}$.

We continue simultaneously for both cases. For $\nu \in\left[1, m_{i}\right]$, we set $V_{\nu}^{(i)}=S_{\nu}^{(i)} A_{\nu}^{(i)}$, with $S_{\nu}^{(i)}, A_{\nu}^{(i)} \in$ $\mathcal{F}\left(G_{i}\right)$ such that $U^{(i)}=S_{1}^{(i)} \cdot \ldots \cdot S_{m_{i}}^{(i)}$. We choose an element $g_{i} \in G_{i}$ with $g_{i} \mid S_{1}^{(i)}$, and define

$$
U=g_{1}^{-1} g_{2}^{-1}\left(g_{1}+g_{2}\right) U^{(1)} U^{(2)} \quad \text { and } \quad V_{1}=g_{1}^{-1} g_{2}^{-1}\left(g_{1}+g_{2}\right) V_{1}^{(1)} V_{1}^{(2)} \text {. }
$$

Then $U, V_{1} \in \mathcal{A}\left(G_{1} \oplus G_{2}\right)$ and $U \mid V_{1} V_{2}^{(1)} \cdot \ldots \cdot V_{m_{1}}^{(1)} V_{2}^{(2)} \cdot \ldots \cdot V_{m_{2}}^{(2)}$, but $U$ divides no proper subproduct. For $W=U^{-1} V_{1} V_{2}^{(1)} \cdot \ldots \cdot V_{m_{1}}^{(1)} V_{2}^{(2)} \cdot \ldots \cdot V_{m_{2}}^{(2)}$ we get

$$
W=U_{2}^{(1)} \cdot \ldots \cdot U_{\ell_{1}}^{(1)} U_{2}^{(2)} \cdot \ldots \cdot U_{\ell_{2}}^{(2)}
$$

and

$$
\mathrm{L}(W)=\mathrm{L}\left(U_{2}^{(1)} \cdot \ldots \cdot U_{\ell_{1}}^{(1)}\right)+\mathrm{L}\left(U_{2}^{(2)} \cdot \ldots \cdot U_{\ell_{2}}^{(2)}\right) .
$$

This show that $\min \mathrm{L}(W)=\left(\ell_{1}-1\right)+\left(\ell_{2}-1\right)$. Summing up we obtain that

$$
\mathrm{t}\left(G_{1} \oplus G_{2}\right) \geq \mathrm{t}\left(G_{1} \oplus G_{2}, U\right) \geq \max \left\{m_{1}+m_{2}, \ell_{1}+\ell_{2}-1\right\}=\ell_{1}+\ell_{2}-1=\mathrm{t}\left(G_{1}\right)+\mathrm{t}\left(G_{2}\right)-1 .
$$

2. Using 1. we infer that

$$
\begin{aligned}
\mathrm{t}\left(G_{1} \oplus G_{2}\right) & \geq \mathrm{t}\left(G_{1}\right)+\mathrm{t}\left(G_{2}\right)-1 \geq \mathrm{D}\left(G_{1}\right)+\mathrm{D}\left(G_{2}\right) \\
& \geq \mathrm{d}^{*}\left(G_{1}\right)+\mathrm{d}^{*}\left(G_{2}\right)+2=\mathrm{d}\left(G_{1} \oplus G_{2}\right)+2=\mathrm{D}\left(G_{1} \oplus G_{2}\right)+1 .
\end{aligned}
$$

For the rest of this section, let $H$ be a reduced Krull monoid, $H \hookrightarrow \mathcal{F}(P)$ a divisor theory with class group $G$ and suppose that every class contains a prime divisor. 
Lemma 4.6. Let $G=C_{2}^{r}$ with $r \geq 3$ and $\ell \in[1, r+1]$. Let $A_{1}, \ldots, A_{\ell}$ be pairwise distinct zerosum free sequences with $\left|A_{i}\right|=r$. Then there exist some $k \in[0, \ell-1]$ and $U_{1}, \ldots, U_{k} \in \mathcal{A}(G)$ with $U_{1} \cdot \ldots \cdot U_{k} \mid A_{1} \cdot \ldots \cdot A_{\ell}$ such that

$$
\left|U_{1} \cdot \ldots \cdot U_{k}\right| \geq 3(\ell-1)
$$

Proof. See [12, Lemma 6.6.5].

Lemma 4.7. Let $G=C_{2}^{r}$ with $r \geq 3, n \geq 3$, and $u, v_{1}, \ldots, v_{n} \in \mathcal{A}(H)$ such that $u \mid v_{1} \cdot \ldots \cdot v_{n}$ and does not divide any proper subproduct. Furthermore, for every $i \in[1, n]$, suppose that $v_{i}=s_{i} a_{i}$ with $a_{i}, s_{i} \in \mathcal{F}(P) \backslash\{1\}$ such that $u=\prod_{i=1}^{n} s_{i}$ and set $w=u^{-1} v_{1} \cdot \ldots \cdot v_{n}$. Then $\min \mathrm{L}(w) \leq \frac{n(r-1)+1}{2}$.

Proof. We set $U=\boldsymbol{\beta}(u), W=\boldsymbol{\beta}(w), V_{i}=\boldsymbol{\beta}\left(v_{i}\right), S_{i}=\widetilde{\boldsymbol{\beta}}\left(s_{i}\right)$ and $A_{i}=\widetilde{\boldsymbol{\beta}}\left(a_{i}\right)$ for all $i \in[1, n]$. It is sufficient to verify the upper bound for $\min \mathrm{L}(W)$. After renumbering if necessary there is some $\ell \in[0, n]$ such that $\left|A_{1}\right|=\ldots=\left|A_{\ell}\right|=r$ and $\left|A_{i}\right| \leq r-1$ for all $i \in[\ell+1, n]$. If $\ell=0$, then $\min \mathrm{L}(W) \leq|W| / 2=n(r-1) / 2$. Let $\ell \geq 1$, and assume to the contrary that there are distinct $i, j \in[1, \ell]$ such that $A_{i}=A_{j}$, say $i=1$ and $j=2$. Then

$$
\sigma\left(S_{1}\right)=\sigma\left(A_{1}\right)=\sigma\left(A_{2}\right)=\sigma\left(S_{2}\right),
$$

hence $u=s_{1} s_{2}$ and $n \leq 2$, a contradiction. Thus $A_{1}, \ldots, A_{\ell}$ are pairwise distinct zero-sum free sequences. By Lemma 4.6 there exist some $k \in[0, \ell-1]$ and $U_{1}, \ldots, U_{k} \in \mathcal{A}(G)$ such that

$$
U_{1} \cdot \ldots \cdot U_{k} \mid A_{1} \cdot \ldots \cdot A_{\ell} \text { and }\left|U_{1} \cdot \ldots \cdot U_{k}\right| \geq 3(\ell-1) .
$$

Setting $W^{\prime}=\left(U_{1} \cdot \ldots \cdot U_{k}\right)^{-1} W$ we infer that

$$
\left|W^{\prime}\right| \leq|W|-3(\ell-1) \leq \ell r+(n-\ell)(r-1)-3(\ell-1)=n(r-1)-2(\ell-1)+1 .
$$

Thus $W$ has a factorization of length at most

$$
k+\frac{\left|W^{\prime}\right|}{2} \leq \frac{1}{2}(n(r-1)+2(k-(\ell-1))+1) \leq \frac{n(r-1)+1}{2} .
$$

Lemma 4.8. Let $G=C_{2}^{r}$ with $r \geq 3$, and suppose that $\mathrm{t}(G) \geq 2+\frac{r(r-1)}{2}$. Then there are $U, V_{1}, \ldots, V_{r+1} \in$ $\mathcal{A}(G)$, where $U \mid V_{1} \cdot \ldots \cdot V_{r+1}$ but $U$ divides no proper subproduct, such that the following properties are satisfied:

(a) $U=e_{1} \cdot \ldots \cdot e_{r+1}$, and $V_{i}=e_{i} A_{i}$ where $A_{i} \in \mathcal{F}(G)$ and $e_{i}=\operatorname{gcd}\left(U, V_{i}\right)$ for all $i \in[1, r+1]$.

(b) $A_{i} A_{j}$ is not zero-sum free for all $i, j \in[1, r+1]$ distinct.

(c) For $W=U^{-1} V_{1} \cdot \ldots \cdot V_{r+1}$ we have $\operatorname{gcd}(U, W)=1$ and $\mathrm{t}(G)=\mathrm{t}(G, U)=1+\min \mathrm{L}(W)$.

Proof. Let $U \in \mathcal{A}(G)$ with $\mathrm{t}(G)=\mathrm{t}(G, U) \geq 2+\frac{r(r-1)}{2}$. Then there are $V_{1}, \ldots, V_{m} \in \mathcal{A}(G)$ with $U \mid V_{1} \cdot \ldots \cdot V_{m}, U \nmid \prod_{i \in I} V_{i}$ for any $I \subsetneq[1, m]$, and such that

$$
\mathrm{t}(G, U)=1+\min \mathrm{L}(W) .
$$

Then Lemma 4.7 (applied with $H=\mathcal{B}(G)$ ) implies that

$$
1+\frac{r(r-1)}{2} \leq \min \mathrm{L}(W) \leq \frac{m(r-1)+1}{2},
$$

and hence $m=r+1=|U|$. Therefore we may assume that Property (a) holds.

Assume to the contrary that Property (b) fails. Then there exist some $i, j \in[1, r+1]$ distinct such that $A_{i} A_{j}$ is zero-sum free, say $i=1$ and $j=2$. We set

$$
\bar{U}=\left(e_{2}+e_{1}\right) \prod_{i=3}^{r+1} e_{i} \text { and } \overline{V_{2}}=\left(e_{2}+e_{1}\right) A_{2} A_{1} .
$$


Then $\bar{U}, \overline{V_{2}} \in \mathcal{A}(G), \bar{U} \mid \overline{V_{2}} V_{3} \cdot \ldots \cdot V_{r+1}$ and

$$
\bar{U}^{-1} \overline{V_{2}} V_{3} \cdot \ldots \cdot V_{r+1}=W=U^{-1} V_{1} \cdot \ldots \cdot V_{r+1} .
$$

Thus Lemma 4.7 implies that $\min \mathrm{L}(W) \leq \frac{r(r-1)}{2}$, a contradiction. Finally we assume to the contrary that Property (c) fails. This means that the set $I \subset[1, r+1]$, defined as

$$
\prod_{i \in I} e_{i}=\operatorname{gcd}(U, W)
$$

is nonempty. Let $i \in I$. Then $e_{i}$ divides $W=A_{1} \cdot \ldots \cdot A_{r+1}$. If $e_{i} \mid A_{i}^{-1} W$, then $U \mid \prod_{j \in[1, r+1] \backslash\{i\}} V_{j}$, a contradiction. Thus $e_{i} \mid A_{i}$ whence $V_{i}=e_{i}^{2}$. If there would exist $i, j \in I$ distinct, then $A_{i} A_{j}=e_{i} e_{j}$ would be zero-sum free. This implies that $|I| \leq 1$.

Since we assumed $I$ to be nonempty, we get that $|I|=1$, say $I=\{r+1\}$. Then for $i \in[1, r]$ we have

$$
V_{i}=e_{i} A_{i} \quad \text { and } \quad V_{r+1}=e_{r+1}^{2} .
$$

After renumbering if necessary, we may suppose that for some $\ell \in[0, r]$ we have $\left|A_{1}\right|=\ldots=\left|A_{\ell}\right|=r$ and $\left|A_{i}\right| \leq r-1$ for all $i \in[\ell+1, r]$. If $\ell=0$, then $\min \mathrm{L}(W) \leq|W| / 2 \leq(1+r(r-1)) / 2$, a contradiction. Suppose that $\ell \geq 1$. By Lemma 4.6 there exists some $k \in[1, \ell-1]$ and $U_{1}, \ldots, U_{k} \in \mathcal{A}(G)$ such that

$$
U_{1} \cdot \ldots \cdot U_{k} \mid A_{1} \cdot \ldots \cdot A_{\ell} \text { and }\left|U_{1} \cdot \ldots \cdot U_{k}\right| \geq 3(\ell-1) .
$$

Setting $W^{\prime}=\left(U_{1} \cdot \ldots \cdot U_{k}\right)^{-1} W$ we infer that

$$
\left|W^{\prime}\right| \leq|W|-3(\ell-1) \leq \ell r+(r-\ell)(r-1)+1-3(\ell-1)=r(r-1)-2 \ell+4 .
$$

Let $W^{\prime}=U_{k+1} \cdot W^{\prime \prime}$ with $U_{k+1} \in \mathcal{A}(G)$ and $e_{r+1} \mid U_{k+1}$. Since $U \nmid \prod_{i=1}^{r} V_{i}, e_{r+1}$ occurs exactly once in $W^{\prime}$ which implies that $\left|U_{k+1}\right| \geq 3$ and $\left|W^{\prime \prime}\right|=\left|W^{\prime}\right|-\left|U_{k+1}\right| \leq r(r-1)-2 \ell+1$. Thus $W$ has a factorization with length at most

$$
k+1+\frac{\left|W^{\prime \prime}\right|}{2}=\frac{1}{2}(r(r-1)+2(k+1-\ell)+1) \leq \frac{r(r-1)}{2}+\frac{1}{2}
$$

whence $\min \mathrm{L}(W) \leq \frac{r(r-1)}{2}$, a contradiction.

Lemma 4.9. Let $G=C_{2}^{r}$ with $r \in \mathbb{N}$.

1. $\mathrm{t}(G)=\mathrm{D}(G)$ if and only if $r \in[2,3]$.

2. If $r=2$, then $\mathrm{t}(H)=\mathrm{D}(G)$.

3. If $r=3$ and if there is a nontrivial class containing at least two distinct prime divisors, then $\mathrm{t}(H)=\mathrm{D}(G)+1$.

Proof. We proceed in four steps, distinguishing the cases $r=1, r=2, r=3$, and $r \geq 4$.

(i) If $r=1$, then $\mathcal{B}(G)$ is factorial and hence $\mathrm{t}(G)=0<\mathrm{D}(G)=2$.

(ii) Suppose that $r=2$. Then $\mathrm{D}(G)=3$, and and by Proposition 3.3 we have

$$
3 \leq \mathrm{t}(H) \leq \max \left\{\mathrm{t}(G),\left\lfloor\frac{3+\left(\mathrm{D}\left(G_{P}\right)-1\right)^{2}}{2}\right\rfloor\right\}=\max \{\mathrm{t}(G), 3\},
$$

and hence it suffices to verify that $\mathrm{t}(G) \leq 3$. This can be done by a quick direct check.

(iii) Suppose that $r=3$. To show the statement on $\mathrm{t}(G)$, we assume to the contrary that $\mathrm{t}(G) \geq$ $\mathrm{D}(G)+1=r+2=5=2+\frac{3 \cdot 2}{2}$. Then let $U, V_{1}, \ldots, V_{5}$ have all the properties of Lemma 4.8, and use all the notations of that lemma. In particular, we have $U=e_{1} \cdot \ldots \cdot e_{4}$. Then $\left(e_{1}, e_{2}, e_{3}\right)$ is a basis of $G$, $e_{4}=e_{1}+e_{2}+e_{3}$, and $G=\left\{0, e_{1}, e_{2}, e_{3}, e_{1}+e_{2}+e_{3}, e_{2}+e_{3}, e_{1}+e_{3}, e_{1}+e_{2}\right\}$. Since $\operatorname{gcd}(U, W)=1$, it follows that

$$
\operatorname{supp}\left(A_{1}\right) \subset \operatorname{supp}(W) \subset\left\{e_{2}+e_{3}, e_{1}+e_{3}, e_{1}+e_{2}\right\} .
$$

On the other hand, $A_{1}$ is zero-sum free with $\sigma\left(A_{1}\right)=e_{1}$ and with $\left|A_{1}\right| \in[2,3]$, a contradiction. 
Now suppose that there is a nontrivial class containing at least two distinct prime divisors. First we show that $\mathrm{t}(H) \geq 5$. Let $\left(e_{1}, e_{2}, e_{3}\right)$ be a basis of $G$ and let $p_{i} \in P \cap e_{i}$ for all $i \in[1,3]$, and let $p_{3}^{\prime} \in P \cap e_{3}$ with $p_{3}^{\prime} \neq p_{3}$. Let $u=q_{1} q_{2} q_{3} p_{3} \in \mathcal{A}(H)$ with $\boldsymbol{\beta}(u)=\left(e_{1}+e_{2}+e_{3}\right)\left(e_{1}+e_{3}\right)\left(e_{2}+e_{3}\right) e_{3}$ such that $q_{1} \in P \cap\left(e_{1}+e_{2}+e_{3}\right), q_{2} \in P \cap\left(e_{1}+e_{3}\right)$, and $q_{3} \in P \cap\left(e_{2}+e_{3}\right)$. Now we define

$$
v_{1}=q_{1} p_{1} p_{2} p_{3}^{\prime}, v_{2}=q_{2} p_{1} p_{3}^{\prime}, v_{3}=q_{3} p_{2} p_{3}^{\prime}, \quad \text { and } \quad v_{4}=p_{3} p_{3} \text {. }
$$

Then $v_{1}, v_{2}, v_{3}, v_{4} \in \mathcal{A}(H), u \mid v_{1} v_{2} v_{3} v_{4}$, but $u$ does not divide any proper subproduct. Since we have $\mathrm{L}\left(u^{-1} v_{1} v_{2} v_{3} v_{4}\right)=\{4\}$, it follows that

$$
\mathrm{t}(H, u) \geq \mathrm{t}(a, u) \geq \max \left\{4,1+\min \mathrm{L}\left(u^{-1} a\right)\right\}=5=\mathrm{D}(G)+1 .
$$

Assume to the contrary that $\mathrm{t}(H)>5$. We choose $a \in H$ and $u \in \mathcal{A}(H)$ such that $\mathrm{t}(H)=\mathrm{t}(a, u) \geq 6$. Then there are $u_{2}, \ldots, u_{\ell}, v_{1}, \ldots, v_{m} \in \mathcal{A}(H)$ such that $u \mid v_{1} \cdot \ldots \cdot v_{m}$, but $u$ divides no proper subproduct, and $\max \{\ell, m\}=\mathrm{t}(a, u)$. We set $U=\boldsymbol{\beta}(u), w=u^{-1} a$, and $W=\boldsymbol{\beta}(W)$. Since $m \leq|U| \leq \mathrm{D}(G)=4$, it follows that $\mathrm{t}(a, u)=\ell=1+\min \mathrm{L}(W) \geq 6$. From this we get that $|U|=4$ and $|W| \in[10,12]$. Then for every $i \in[1,4]$, there are $p_{i} \in P$ and $a_{i} \in F \backslash\{1\}$ such that $v_{i}=p_{i} a_{i}$ and $u=p_{1} p_{2} p_{3} p_{4}$. We set $A_{i}=\boldsymbol{\beta}\left(a_{i}\right)$ for all $i \in[1,4]$, and after renumbering if necessary there is an $s \in[0,4]$ such that $\left|A_{1}\right|=\ldots=\left|A_{s}\right|=3$, and $3>\left|A_{s+1}\right| \geq \ldots \geq\left|A_{4}\right|$. Note that $W=A_{1} \ldots A_{4}$, and since $\sigma\left(A_{1}\right), \ldots, \sigma\left(A_{4}\right)$ are pairwise distinct, the sequences $A_{1}, \ldots, A_{4}$ are pairwise distinct. Since $\left|A_{1} \cdot \ldots \cdot A_{4}\right|=|W| \in[10,12],|W|=10$ implies $s \geq 2,|W|=11$ implies $s \geq 3$, and $|W|=12$ implies $s=4$. By Lemma 4.6 there exist $k \in[0, s-1]$ and $W_{1}, \ldots, W_{k} \in \mathcal{A}(G)$ such that $W_{1} \cdot \ldots \cdot W_{k} \mid A_{1} \cdot \ldots \cdot A_{s}$ and $\left|W_{1} \cdot \ldots \cdot W_{k}\right| \geq 3(s-1)$. This implies that

$$
5 \leq \min \mathrm{L}(W) \leq k+\frac{|W|-\left|W_{1} \cdot \ldots \cdot W_{k}\right|}{2} \leq(s-1)+\frac{|W|-3(s-1)}{2}=\frac{|W|-(s-1)}{2},
$$

a contradiction.

(iv) Suppose that $r \geq 4$. If $r \geq 4$ is even, then Lemma 4.3 , 2 shows that

$$
\mathrm{t}(G) \geq 1+\frac{r^{2}}{2}>r+1=\mathrm{D}(G)
$$

If $r \geq 5$ is odd, then again by Lemma 4.3,2 we get that

$$
\mathrm{t}(G) \geq \mathrm{t}\left(C_{2}^{r-1}\right) \geq 1+\frac{(r-1)^{2}}{2}>r+1=\mathrm{D}(G) .
$$

Lemma 4.10. Let $G=C_{3}^{r}$ with $r \in \mathbb{N}$. Then $\mathrm{t}(H)=\mathrm{D}(G)$ if and only if $r=1$.

Proof. Let $r=1$. Then $\mathrm{D}(G)=3$, and by Proposition 3.3 we have

$$
3 \leq \mathrm{t}(H) \leq \max \left\{\mathrm{t}(G),\left\lfloor\frac{3+\left(\mathrm{D}\left(G_{P}\right)-1\right)^{2}}{2}\right\rfloor\right\}=\max \{\mathrm{t}(G), 3\},
$$

and hence it suffices to check that $\mathrm{t}(G) \leq 3$. Let $U \in \mathcal{A}(G)$. If $|U|=2$, then $\mathrm{t}(G, U) \leq 1+\frac{|U|(\mathrm{D}(G)-1)}{2}=3$. If $|U|=3$, then $U=g^{3}, V_{1}=V_{2}=V_{3}=(-g) g$ for some nonzero $g \in G$, and hence $\mathrm{t}(G, U)=2$.

Note that $\mathrm{D}\left(C_{3}^{r}\right)=\mathrm{D}^{*}\left(C_{3}^{r}\right)=2 r+1$. Since $\mathrm{t}(H) \geq \mathrm{t}(G)$, Proposition 4.5 implies that it is sufficient to show that $\mathrm{t}\left(C_{3} \oplus C_{3}\right)>5=\mathrm{D}\left(C_{3} \oplus C_{3}\right)$.

Let $G=C_{3} \oplus C_{3}$, and let $\left(e_{1}, e_{2}\right)$ be a basis of $G$. We define $V_{1}=V_{2}=e_{1}\left(-e_{1}-e_{2}\right)^{2}\left(e_{2}-e_{1}\right)^{2}, V_{3}=V_{4}=e_{2}\left(-e_{1}-e_{2}\right)^{2}\left(e_{1}-e_{2}\right)^{2}, V_{5}=\left(e_{1}+e_{2}\right)\left(-e_{1}\right)^{2}\left(e_{1}-e_{2}\right)$, and

$$
U=e_{1}^{2} e_{2}^{2}\left(e_{1}+e_{2}\right)
$$

Then $U, V_{1}, \ldots, V_{5} \in \mathcal{A}(G), U \mid V_{1} \cdot \ldots \cdot V_{5}$, but $U$ does not divide any proper subproduct. We set $W=U^{-1} V_{1} \cdot \ldots \cdot V_{5}$, and assert that $\min \mathrm{L}(W)=6$, which implies that

$$
\mathrm{t}(G) \geq \mathrm{t}(G, U) \geq 1+\min \mathrm{L}(W)=7 .
$$


Note $W=\left(-e_{1}-e_{2}\right)^{8}\left(e_{1}-e_{2}\right)^{5}\left(e_{2}-e_{1}\right)^{4}\left(-e_{1}\right)^{2}$, in particular is has lenght 19 . We determine the atoms $S \in \mathcal{A}(G)$ with $S \mid W$ and $|S| \geq 4$. Such an atom must not contain both $\left(e_{1}-e_{2}\right)$ and $\left(e_{2}-e_{1}\right)$, yet contains at least three distinct elements; consequently it contains $-e_{1}$. First, suppose the two elements besides $-e_{1}$ are $\left(-e_{1}-e_{2}\right)$ and $\left(e_{2}-e_{1}\right)$. We note that $\left(-e_{1}\right)\left(-e_{1}-e_{2}\right)\left(e_{2}-e_{1}\right)$ is a (minimal) zero-sum sequence, and thus the only minimal zero-sum sequence with this support. Thus, since $|S| \geq 4$, we have $\operatorname{supp}(S)=\left\{-e_{1},-e_{1}-e_{2}, e_{1}-e_{2}\right\}$. If the multiplicity of $-e_{1}$ is 1 , we get the atom $\left(-e_{1}\right)\left(-e_{1}-e_{2}\right)\left(e_{1}-e_{2}\right)^{2}$, and if the this multiplicity is 2 , we get the atom $\left(-e_{1}\right)^{2}\left(-e_{1}-e_{2}\right)^{2}\left(e_{1}-e_{2}\right)$.

Therefore, noting that the multiplicty of $-e_{1}$ in $W$ is 2 , we can infer that every factoriazation of $W$ contains (counted with multiplicity) either one atom of lengths 5 and none of lengths 4 or none of length 5 and at most 2 of length 4 . Thus, $\min \mathrm{L}(W)$ is at least the smaller of $1+\lceil(19-5) / 3\rceil=6$ and $2+\lceil(19-2 \cdot 4) / 3=6$; that is it is at least 6 .

Lemma 4.11. Let $G=C_{4}^{r}$ with $r \in \mathbb{N}$. Then $\mathrm{t}(H)=\mathrm{D}(G)$ if and only if $r=1$.

Proof. Let $r=1$. Let $u \in \mathcal{A}(H)$ but not prime. We have to show that $\mathrm{t}(H, u) \leq \mathrm{D}(G)=4$. Let $v_{1}, \ldots, v_{m} \in \mathcal{A}(H)$ such that $u \mid v_{1} \ldots \cdot v_{m}$, but $u$ divides no proper subproduct. We set $w=u^{-1} v_{1} \cdot \ldots \cdot v_{m} \in$ $H$, and note that $m \leq|u|$ and $m \in[2,4]$. If $m=2$, then $\left|u^{-1} v_{1} v_{2}\right| \leq 6$, and hence $\min \mathrm{L}(w) \leq 3$. We set $U=\boldsymbol{\beta}(u), W=\boldsymbol{\beta}(w), V_{i}=\boldsymbol{\beta}\left(v_{i}\right)$ for all $i \in[1, m]$, and distinguish the cases $m=3$ and $m=4$.

CASE 1: $m=3$.

First suppose that $|U|=3$. Then $U=g^{2}(2 g)$ for some $g \in G$ with $\operatorname{ord}(g)=4$, and we may suppose that $g\left|V_{1}, g\right| V_{2}$, and $(2 g) \mid V_{3}$. Then $\left|V_{3}\right| \leq 3$. Assume to the contrary that $\min \mathrm{L}(W) \geq 4$. Then $|W| \geq 8$, which implies that $\left|V_{1}\right|=\left|V_{2}\right|=4$ and $\left|V_{3}\right|=3$. Then $V_{1}=V_{2}=g^{4}$, and $V_{3} \in\left\{(2 g) g^{2},(2 g)(-g)^{2}\right\}$. In both cases we get that $\min \mathrm{L}(W)<4$, a contradiction.

Now suppose that $|U|=4$. Then $U=g^{4}$ for some $g \in G$ with $\operatorname{ord}(g)=4$, and we assume again that $\min \mathrm{L}(W) \geq 4$. This implies that $|W|=8$, and hence, after renumbering if necessary, $V_{1} \in\left\{g^{4}, g^{2}(2 g)\right\}$, and $V_{2}=V_{3}=g^{4}$. Thus we get $\mathrm{L}(W)=\{2\}$, a contradiction.

CASE 2: $m=4$.

Then $U=g^{4}$ for some $g \in G$ with ord $(g)=4$. Thus $V_{1}, V_{2}, V_{3}, V_{4} \in\left\{g(-g), g^{2}(2 g), g^{4}\right\}$. Assume to the contrary that $\min \mathrm{L}(W) \geq 4$. Then $|W| \geq 8$, and at most two of the $V_{i}$ are equal to $(-g) g$. Discussing all possibilities we quickly see that $\min \mathrm{L}(W) \leq 3$, a contradiction.

Now suppose that $r \geq 2$, and note that $\mathrm{D}\left(C_{4}^{r}\right)=\mathrm{D}^{*}\left(C_{4}^{r}\right)=3 r+1$. Since $\mathrm{t}(H) \geq \mathrm{t}(G)$, it suffices to prove that $\mathrm{t}(G)>\mathrm{D}(G)$. Thus by Proposition 4.5 it is sufficient to show that $\mathrm{t}\left(C_{4} \oplus C_{4}\right)>7=\mathrm{D}\left(C_{4} \oplus C_{4}\right)$.

Let $G=C_{4} \oplus C_{4}$ and let $\left(e_{1}, e_{2}\right)$ be a basis of $G$. We define

$$
\begin{aligned}
V_{1}=V_{2}=V_{3} & =e_{1}\left(-e_{1}-e_{2}\right)^{3}\left(2 e_{1}-e_{2}\right), \\
V_{4}=V_{5}=V_{6} & =e_{2}\left(-e_{1}-e_{2}\right)^{3}\left(-e_{1}+2 e_{2}\right), \\
V_{7} & =\left(e_{1}+e_{2}\right)^{4}, \quad \text { and } \quad U=e_{1}^{3} e_{2}^{3}\left(e_{1}+e_{2}\right) .
\end{aligned}
$$

Then $U, V_{1}, \ldots, V_{7} \in \mathcal{A}(G), U \mid V_{1} \cdot \ldots \cdot V_{7}$, but $U$ does not divide any proper subproduct. We set $W=U^{-1} V_{1} \cdot \ldots \cdot V_{7}$, and assert that $\min \mathrm{L}(W)=7$, which implies that

$$
\mathrm{t}(G) \geq \mathrm{t}(G, U) \geq 1+\min \mathrm{L}(W)=8 .
$$

First we determine the atoms $S \in \mathcal{A}(G)$ with $S \mid W$. Since

$$
\left(2 e_{1}-e_{2},-e_{1}+2 e_{2}\right)=\left(e_{1}, e_{2}\right)\left(\begin{array}{cc}
2 & -1 \\
-1 & 2
\end{array}\right)
$$

and the determinant of the transformation matrix equals -1 modulo 4 , it follows that $\left(2 e_{1}-e_{2},-e_{1}+2 e_{2}\right)$ is independent, and hence the sequence $\left(2 e_{1}-e_{2}\right)^{3}\left(-e_{1}+2 e_{2}\right)^{3}$ is zero-sum free. Now it is easy to check that

$S_{1}=\left(e_{1}+e_{2}\right)\left(2 e_{1}-e_{2}\right)^{3}\left(-e_{1}+2 e_{2}\right)^{3}, S_{2}=\left(e_{1}+e_{2}\right)^{2}\left(2 e_{1}-e_{2}\right)^{2}\left(-e_{1}+2 e_{2}\right)^{2}, S_{3}=\left(e_{1}+e_{2}\right)^{3}\left(2 e_{1}-e_{2}\right)\left(-e_{1}+2 e_{2}\right)$ 
are the atoms $S$ with $\left(-e_{1}-e_{2}\right) \nmid S$ and $S \mid W$, and

$$
S_{4}=\left(-e_{1}-e_{2}\right)\left(2 e_{1}-e_{2}\right)\left(-e_{1}+2 e_{2}\right), S_{5}=\left(-e_{1}-e_{2}\right)\left(e_{1}+e_{2}\right), S_{6}=\left(-e_{1}-e_{2}\right)^{4}
$$

are the atoms $S$ with $\left(-e_{1}-e_{2}\right)|S| W$. We claim that

$$
\mathrm{Z}(W)=\left\{S_{1} S_{5}^{2} S_{6}^{4}, S_{3} S_{4}^{2} S_{6}^{4}, S_{2} S_{4} S_{5} S_{6}^{4}, S_{4}^{3} S_{5}^{3} S_{6}^{3}\right\}
$$

which implies that $\mathrm{L}(W)=\{7,9\}$. Clearly, it remains to show that the given factorizations are the only ones. Let $z \in \mathrm{Z}(W)$. If $S_{1} \mid z$, then obviously $z=S_{1} S_{5}^{2} S_{6}^{4}$. Suppose that $S_{1} \nmid z$. If $S_{3} \mid z$, then $z=S_{3} S_{4}^{2} S_{6}^{4}$. Suppose that $S_{3} \nmid z$. If $S_{2} \mid z$, then $z=S_{2} S_{4} S_{5} S_{6}^{4}$. If also $S_{2} \nmid z$, then $z=S_{4}^{3} S_{5}^{3} S_{6}^{3}$.

Theorem 4.12. Let $H$ be a Krull monoid with finite class group $G$ such that every class contains a prime divisor.

1. $\mathrm{t}(G)=\mathrm{D}^{*}(G)$ if and only if $G \in\left\{C_{3}, C_{4}, C_{2}^{2}, C_{2}^{3}\right\}$.

2. If one nontrivial class contains at least two distinct prime divisors, then $\mathrm{t}(H)=\mathrm{D}^{*}(G)$ if and only if $G \in\left\{C_{2}, C_{3}, C_{4}, C_{2}^{2}\right\}$.

3. Suppose that $G$ has rank $\mathrm{r}(G)=r$, and consider both, $\mathrm{D}(G)$ and $\mathrm{t}(H)$, as functions in $r$. Then there are constants $M_{1}, M_{2}, M_{3}, M_{4} \in \mathbb{R}_{>0}$ (depending only on $\exp (G)$ but not on $r$ ) such that

$$
M_{1} r \leq \mathrm{D}(G) \leq M_{2} r \quad \text { and } \quad M_{3} r^{2} \leq \mathrm{t}(H) \leq M_{4} r^{2} .
$$

In particular, $\mathrm{t}(H)$ grows as the upper bound $1+\mathrm{D}(G)(\mathrm{D}(G)-1) / 2$, given in Proposition 3.3 .3.

Proof. If $|G|=1$, then both, $H$ and $\mathcal{B}(G)$, are factorial, whence $\mathrm{t}(H)=\mathrm{t}(G)=0$, but we have $\mathrm{D}^{*}(G)=1$. From now on we suppose that $|G|>1$, say $G \cong C_{n_{1}} \oplus \cdots \oplus C_{n_{r}}$, where $r=\mathrm{r}(G) \in \mathbb{N}$ is the rank of $G$, $n_{1}, \ldots, n_{r} \in \mathbb{N}, 1<n_{1}|\ldots| n_{r}$, and $n=n_{r}=\exp (G)$ is the exponent of $G$.

1. If $|G|=2$, then $\mathrm{t}(G)=0$ and $\mathrm{D}^{*}(G)=2$. Suppose that $|G|>2$, and that $\mathrm{t}(G)=\mathrm{D}^{*}(G)$. By Lemma 4.1 we obtain that $\mathrm{t}(G) \geq 1+\exp (G) \mathrm{k}^{*}(G)$. Therefore Lemma 4.4 implies that equality holds and that $G$ is a $p$-group with $n_{1}=\ldots=n_{r}$, whence $G=C_{n}^{r}$. If $n \geq 5$, then $\mathrm{t}\left(C_{n}\right)>n=\mathrm{D}\left(C_{n}\right)$ by Lemma 4.12. Thus Proposition 4.5.2 implies that $\mathrm{t}\left(C_{n}^{r}\right)>\mathrm{D}\left(C_{n}^{r}\right)$. Therefore it remains to consider the cases where $n \in[2,4]$. Lemmas 4.9 4.10, and 4.11 show that the mentioned groups satisfy $\mathrm{t}(G)=\mathrm{D}^{*}(G)$, and that there are no other groups $G$ with $\exp (G) \leq 4$ with this property.

2. If $|G|=2$, then $H$ is not factorial whence $\mathrm{t}(H) \geq 2$, and Proposition 3.3 .3 implies that $\mathrm{t}(H) \leq 2$. If $G=C_{2}^{3}$, then Lemma 4.9 implies that $\mathrm{t}(H)=\mathrm{D}(G)+1$. Since $\mathrm{t}(H) \geq \mathrm{t}(G) \geq \mathrm{D}(G) \geq \mathrm{D}^{*}(G)$, the remaining assertions follow from 1 .

3. We have $1+r\left(n_{1}-1\right) \leq \mathrm{D}^{*}(G) \leq \mathrm{D}(G) \leq \mathrm{D}\left(C_{n}^{r}\right)$, and by [12, Theorem 5.5.5], we obtain that

$$
\mathrm{D}\left(C_{n}^{r}\right) \leq n+n \log n^{r-1} \leq(n \log n) r .
$$

Thus there exist constants $M_{1}, M_{2}$ as required. Let $p$ be a prime with $p \mid n_{1}$. Then $G$ has a subgroup isomorphic to $C_{p}^{r}$ and hence $\mathrm{t}\left(C_{p}^{r}\right) \leq \mathrm{t}(G)$. We intend to find a $M_{3} \in \mathbb{R}_{>0}$ with $M_{3} r^{2} \leq \mathrm{t}\left(C_{p}^{r}\right)$. If $p=2$, this holds by Lemma 4.3.2. Let $p$ be odd. Then there is an $s \in[r-2, r]$ such that $s$ is even and $\operatorname{gcd}(p, s-1)=1$, and hence Lemma 4.3.2 implies that

$$
1+2 s\left\lfloor\frac{s}{2 p}\right\rfloor \leq \mathrm{t}\left(C_{p}^{s}\right) \leq \mathrm{t}\left(C_{p}^{r}\right) .
$$

Finally Proposition 3.33 implies that

$$
\mathrm{t}(H) \leq 1+\frac{\mathrm{D}(G)(\mathrm{D}(G)-1)}{2} \leq \mathrm{D}(G)^{2} \leq M_{2}^{2} r^{2} .
$$




\section{KRULL MONOIDS WHOSE CLASS GROUP IS EITHER CYCLIC OR AN ELEMENTARY 2-GROUP}

In this section we study Krull monoids $H$ whose class group $G$ is either cyclic or an elementary 2-group. We get quite precise results, which confirm the general tendency of the tame degree indicated by Theorem 4.12. Suppose $|G| \geq 3$, that every class contains a prime divisor, and consider again the inequality

$$
\mathrm{D}(G) \leq \mathrm{t}(H) \leq 1+\frac{\mathrm{D}(G)(\mathrm{D}(G)-1)}{2} .
$$

In case of elementary 2-groups, $\mathrm{t}(H)$ almost equals the upper bound and, apart from one exceptional case, we always have $\mathrm{t}(H)=\mathrm{t}(G)$. Suppose that $G$ is cyclic of order $|G|=n \geq 5$. Then $\mathrm{D}(G)=n \leq \mathrm{t}(H) \leq n^{2}$ (for better lower bounds see Lemma 4.1). As expected, it turns out that the tame degree is close to the lower bound.

Theorem 5.1. Let $H$ be a Krull monoid whose class group $G$ is an elementary 2-group, say $G \cong C_{2}^{r}$ with $r \in \mathbb{N}$, and suppose that every class contains a prime divisor. Then we have

1. If $r=1$, then $\mathrm{t}(H)=2$ and $\mathrm{t}(G)=0$.

2. If $r=3$, then $\mathrm{t}(G)=4$, and if one nontrivial class contains at least two distinct prime divisors, then $\mathrm{t}(H)=5$.

3 .

$$
\mathrm{t}(H)=\mathrm{t}(G) \quad \begin{cases}=1+\frac{r^{2}}{2} & \text { if } r \geq 2 \quad \text { is even, } \\ \geq 2+\frac{r(r-1)}{2} & \text { if } r \geq 5 \quad \text { is odd. }\end{cases}
$$

Proof. We may suppose that $H$ is reduced, and that $H \hookrightarrow \mathcal{F}(P)$ a divisor theory with class group $G$. All statements of 1. and 2. follow from Lemma 4.9 and from Theorem 4.12 and hence it remains to prove 3. The assertion for even $r \geq 2$ follows from [12, Corollary 6.5.6]. Suppose that $r \geq 5$ is odd. The lower bound for $\mathrm{t}(G)$ follows from [12, Theorem 6.5.3]. So it remains to show that $\mathrm{t}(H)=\mathrm{t}(G)$. By Proposition 3.3, it suffices to show that $\mathrm{t}(H) \leq \mathrm{t}(G)$.

Let $u \in \mathcal{A}(H)$. We have to show that $\mathrm{t}(H, u) \leq \mathrm{t}(G)$. If $u \in P$, then $\mathrm{t}(H, u)=0$. Suppose that $u \notin P$, and let $a \in u H, v_{1}, \ldots, v_{n} \in \mathcal{A}(H)$ with $z=v_{1} \cdot \ldots \cdot v_{n} \in \mathbf{Z}(a)$ such that $u$ divides no proper subproduct of $v_{1} \cdot \ldots \cdot v_{n}$, and $\operatorname{such}$ that $\mathrm{t}(H, u)=\max \{n, 1+\min \mathrm{L}(w)\}$, where $w=u^{-1} v_{1} \cdot \ldots \cdot v_{n}$. If $n \leq 2$, then the assertion follows. Suppose that $n \geq 3$, and note that $n \leq|u|$. Then Lemma 4.7 implies that $\min \mathrm{L}(w) \leq \frac{n(r-1)+1}{2}$. Thus we are done for $n \leq r$.

Suppose that $n=r+1$. Then $u=p_{1} \cdot \ldots \cdot p_{r+1}$ and, for all $i \in[1, r+1], v_{i}=p_{i} a_{i}$ where $p_{i} \in P$ and $a_{i} \in \mathcal{F}(P) \backslash\{1\}$. For $i \in[1, r+1]$, we set $V_{i}=\boldsymbol{\beta}\left(v_{i}\right), g_{i}=\left[p_{i}\right]$ and we set $U=\boldsymbol{\beta}(u)$. Then $U \mid V_{1} \cdot \ldots \cdot V_{r+1}$. After renumbering if necessary we may assume that there is some $m \in[1, r+1]$ such that $U \mid V_{1} \cdot \ldots \cdot V_{m}$ but $U$ does not divide any proper subproduct.

If $m=1$, then $V_{1}=U, \overline{\boldsymbol{\beta}}(z)=V_{1} \cdot \ldots \cdot V_{r+1} \in U \mathrm{Z}(G)$, and by definition of the tame degree in the fibres, there is a $z^{\prime} \in \mathrm{Z}(a) \cap u \mathrm{Z}(H)$ with $\overline{\boldsymbol{\beta}}\left(z^{\prime}\right)=\overline{\boldsymbol{\beta}}(z)$ and $\mathrm{d}\left(z, z^{\prime}\right) \leq \mathrm{t}(H, u, \boldsymbol{\beta})$. By Lemma 3.2, we get $\mathrm{t}(H, u, \boldsymbol{\beta}) \leq 1+\mathrm{D}(G)=r+2 \leq \mathrm{t}(G)$.

Suppose that $m \geq 2$. There exist $U_{2}, \ldots, U_{k} \in \mathcal{A}(G)$ such that $V_{1} \cdot \ldots \cdot V_{m}=U U_{2} \cdot \ldots \cdot U_{k}$ and

$$
\max \{k, m\}=\mathrm{d}\left(V_{1} \cdot \ldots \cdot V_{m}, U U_{2} \cdot \ldots \cdot U_{k}\right) \leq \mathrm{t}(G, U) \leq \mathrm{t}(G) .
$$

Since $\boldsymbol{\beta}\left(u^{-1} a\right)=U_{2} \cdot \ldots \cdot U_{k} V_{m+1} \cdot \ldots \cdot V_{r+1}$ and $\boldsymbol{\beta}: H \rightarrow \mathcal{B}(G)$ is a transfer homomorphism, there exist $u_{2}, \ldots, u_{k}, w_{m+1}, \ldots, w_{r+1} \in \mathcal{A}(H)$ such that $\boldsymbol{\beta}\left(u_{i}\right)=U_{i}$ for all $i \in[2, k], \quad \boldsymbol{\beta}\left(w_{j}\right)=V_{j}$ for all $j \in[m+1, r+1]$ and $u^{-1} a=u_{2} \cdot \ldots \cdot u_{k} w_{m+1} \cdot \ldots \cdot w_{r+1}$. Then

$$
z^{\prime}=u u_{2} \cdot \ldots \cdot u_{k} w_{m+1} \cdot \ldots \cdot w_{r+1} \in \mathbf{Z}(a) \cap u \mathbf{Z}(H) \quad \text { and } \quad \mathrm{d}\left(z, z^{\prime}\right) \leq \max \{r+1, k+r+1-m\} .
$$

If $m=r+1$, this implies $\mathrm{d}\left(z, z^{\prime}\right) \leq \mathrm{t}(G)$. If $m=2$, then

$$
k-1 \leq\left\lfloor\frac{\left|V_{1} V_{2}\right|-|U|}{2}\right\rfloor \leq\left\lfloor\frac{2 \mathrm{D}(G)-3}{2}\right\rfloor=r-1,
$$


and

$$
k+1+r-m \leq 2 r-1 \leq 2+\frac{r(r-1)}{2} \leq \mathrm{t}(G) .
$$

Suppose that $m \in[3, r]$. Then Lemma 4.7 (applied with $H=\mathcal{B}(G)$ and $n=m \geq 3$ ) implies that $k-1 \leq\left\lfloor\frac{m(r-1)+1}{2}\right\rfloor$. If $m \leq r-1$, then

$$
k+r+1-m \leq 2+\frac{m(r-1)+1}{2}+r-m \leq 2+\frac{r(r-1)}{2} \leq \mathrm{t}(G),
$$

If $m=r$, then $k-1 \leq \frac{r(r-1)}{2}$ and

$$
k+r+1-m=k+1 \leq 2+\frac{r(r-1)}{2} \leq \mathrm{t}(G) .
$$

Thus in both cases we get $\mathrm{d}\left(z, z^{\prime}\right) \leq \mathrm{t}(G)$.

For $m, n \in \mathbb{N}$, let $\omega(n)$ denote the number of distinct prime divisors of $n$, and let $\phi_{m}(n)$ denote the number of integers $a \in[1, m]$ with $\operatorname{gcd}(a, n)=1$; this function is sometimes called Legendre's totient function. Thus $\phi_{n}(n)=\phi(n)$ is Euler's totient function.

Theorem 5.2. Let $H$ be a Krull monoid having a cyclic class group $G$ of order $|G|=n \geq 5$.

1. If $n=p \in \mathbb{P}$, then $\mathrm{t}(H) \leq 1+\frac{2(p-1) p}{p+5}+2(p-1)\left(\frac{1}{2}+\log \left(\frac{p+3}{2}\right)\right)$.

2. If $n=p^{\alpha}$, where $p \in \mathbb{P}$ and $\alpha \geq 2$, then

$$
\mathrm{t}(H) \leq 1-2 \alpha+\frac{2 p^{\alpha+1}}{p-1}+2 \alpha n+3 \sum_{i=1}^{\alpha}\left(p^{i}-1\right) \log \frac{p^{i}}{2} .
$$

3. If $n=p_{1}^{\alpha_{1}} \cdot \ldots \cdot p_{r}^{\alpha_{r}}$, where $r \geq 2, \alpha_{1}, \ldots, \alpha_{r} \in \mathbb{N}$, and $p_{1}, \ldots, p_{r} \in \mathbb{P}$ are distinct, then $\mathrm{t}(H) \leq$ $1+\frac{4.3}{2} \sum_{1<d \mid n}(d-1)+n \sum_{1<d \mid n, d \leq 4375} \frac{d}{2}+n \sum_{4376 \leq d \mid n} 2^{\omega(d)+1} \sqrt{2 \omega(d)}+3.3 \sum_{1<d \mid n}(d-1) \log \left(\left\lfloor\frac{d+1}{2^{\omega(d)+1} \sqrt{2 \omega(d)-1}+1}-1\right\rfloor\right)$.

We need a new combinatorial invariant and a series of lemmas. The proof of Theorem 5.2 will be given at the end of this section.

Definition 5.3. Let $G$ be a finite abelian group. For every $t \in[2, \mathrm{D}(G)]$, let $\mathrm{m}(G, t)$ denote the smallest integer $\ell \in \mathbb{N}$ such that every sequence $S$ over $G \backslash\{0\}$ of length $|S| \geq \ell$ and in addition satisfying $\vee_{g}(S) \leq \operatorname{ord}(g)$ for each $g \in G \backslash\{0\}$, has a minimal zero-sum subsequence $T$ of length $|T| \geq t$.

The idea behind defining this constant is to somehow quantify how easy or hard it is for a given group $G$ to avoid the existence of long minimal zero-sum subsequences. While it is clear that some additional condition, beyond the usual one on the length, is needed to make this definition a meaningful one, regarding the precise condition there is some flexibility. The one we choose is, except for excluding 0 , the most permissive one that seems reasonable. If one cares about minimal zero-sum sequences one never has a need for an element more than its order times. To exclude 0 makes sense for the present application and more generally is convenient; the variant of the constant where 0 would be admitted (with multiplicity 1) would merely differ by exactly 1 from the current version.

The following lemma establishes some basic properties of this new invariant.

Lemma 5.4. Let $G$ be a finite abelian group with $|G|>1$.

1. For every $t \in[2, \mathrm{D}(G)]$, we have $\mathrm{m}(G, t) \geq \mathrm{D}(G)$.

2. We have $\mathrm{m}(G, 2)=\mathrm{D}(G)$ and $\mathrm{m}(G, 3) \in\left[2 \mathrm{D}^{*}(G)-1,2 \mathrm{D}(G)-1\right]$. 
Proof. 1. By definition of $\mathrm{D}(G)$, there is a zero-sum free sequence $S$ over $G$ of length $|S|=\mathrm{D}(G)-1$; note that, $S$ being zero-sum free, $\mathrm{v}_{g}(S) \leq \operatorname{ord}(g)$ for each $g$. Since such a sequence does not satisfy the defining property of $\mathrm{m}(G, t)$, it follows that $\mathrm{m}(G, t) \geq \mathrm{D}(G)$ for every $t \in[2, \mathrm{D}(G)]$.

2. Every sequence $S$ over $G \backslash\{0\}$ of length $\mathrm{D}(G)$ has a zero-sum subsequence, and hence a minimal zero-sum subsequence $T$. Since $0 \nmid S$, we get $|T| \geq 2$. Thus $\mathrm{m}(G, 2) \leq \mathrm{D}(G)$, and equality follows by 1 .

In order to show that $\mathrm{m}(G, 3) \leq 2 \mathrm{D}(G)-1$, let $S$ be a sequence of length $|S| \geq 2 \mathrm{D}(G)-1$. We write $S$ in the form $S=S_{1} S_{2} T_{1} T_{2}$ where, for $i \in[1,2], S_{i}$ is a sequence over $G$ such that $\operatorname{supp}\left(S_{i}\right) \cap \operatorname{supp}\left(-S_{i}\right)=\emptyset$ and $T_{i}$ is a squarefree sequence over $G$ containing only elements of order 2 . (Recall that the multiplicity of an element of order 2 in $S$ is at most 2, and also note that $S_{1} S_{2}$ cannot contain elements of order 2 appearing in $S$.)

Without restriction we may suppose that $\left|S_{1}\right| \geq\left|S_{2}\right|$ and $\left|T_{1}\right| \geq\left|T_{2}\right|$. Then $\left|S_{1} T_{1}\right| \geq \mathrm{D}(G)$, and $S_{1} T_{1}$ thus contains a minimal zero-sum subsequence $T$. By construction we have $|T| \geq 3$.

Finally, we verify that $\mathrm{m}(G, 3) \geq 2 \mathrm{D}^{*}(G)-1$. Suppose that $G=C_{n_{1}} \oplus \ldots \oplus C_{n_{r}}$ with $1<n_{1}|\ldots| n_{r}$, and let $\left(e_{1}, \ldots, e_{r}\right)$ be a basis of $G$ with $\operatorname{ord}\left(e_{i}\right)=n_{i}$ for all $i \in[1, r]$. Then

$$
S=\prod_{i=1}^{r} e_{i}^{n_{i}-1}
$$

is a zero-sum free sequence of length $|S|=\mathrm{D}^{*}(G)-1$, and the sequence $(-S) S$ fulfills the additional condition (for slightly different reasons in the cases ord $\left(e_{i}\right)=2$ and $\operatorname{ord}\left(e_{i}\right)>2$ ), and has no minimal zero-sum subsequence $T$ of length $|T| \geq 3$. Thus it follows that $\mathrm{m}(G, 3)>|(-S) S|=2 \mathrm{D}^{*}(G)-2$.

From now on till the rest of this section, let $G$ be a finite cyclic group of order $|G|=n \geq 5, G^{\bullet}=G \backslash\{0\}$, and $m \in[1, n]$.

Lemma 5.5. Let $S$ be a sequence over $G$ such that $\operatorname{ord}(g)=n$ for all $g \in \operatorname{supp}(S)$. If $|S| \geq \frac{\phi(n)(n-1)+1}{\phi_{m}(n)}$, then $S$ has a minimal zero-sum subsequence of length at least $\left\lceil\frac{n}{m}\right\rceil$.

Proof. For $e \in G$ with $\operatorname{ord}(e)=n$, we have $S=m_{1}^{e} e \cdot \ldots \cdot m_{k}^{e} e$ where $m_{1}^{e}, \ldots, m_{k}^{e} \in[1, n]$. For every $\ell \in[2, n-1]$, let $S(\ell, e)$ denote the subsequence consisting of all terms $m_{k}^{e} e$ with $m_{k}^{e} \in[1, \ell]$. Clearly, if

$$
|S(\ell, e)| \geq n
$$

then $S(\ell, e)$ has a minimal zero-sum subsequence of length at least $\left\lceil\frac{n}{\ell}\right\rceil$. So, it suffices to prove the existence of some $e$ such that (5.1) holds with $\ell=m$. Since $\operatorname{ord}(g)=n$ for each $g \mid S$ we have

$$
\sum_{e \in G, \operatorname{ord}(e)=n} S(m, e)=|S| \sum_{i \in[1, m], \operatorname{gcd}(i, n)=1} 1=\phi_{m}(n)|S| .
$$

Therefore,

$$
\max \{|S(m, e)| \mid e \in G, \operatorname{ord}(e)=n\} \geq \frac{\phi_{m}(n)|S|}{\phi(n)} .
$$

It follows from $|S| \geq \frac{\phi(n)(n-1)+1}{\phi_{m}(n)}$ that $\max \{|S(m, e)| \mid e \in G, \operatorname{ord}(e)=n\}>n-1$. This proves (5.1) holds for some $e$ and completes the proof.

The following technical lemma establishes some bounds on sums that is needed several times later on; the somewhat unusual indexing is convenient then.

Lemma 5.6. Let $c_{2}, \ldots, c_{M}$ be non-negative reals. Let $C$ be such that $\sum_{i=2}^{m} i c_{i} \leq C m$ for each $m \in[2, M]$. Then $\sum_{i=2}^{m} c_{i} \leq C\left(1+\sum_{i=3}^{m} 1 / i\right)$ for each $m \in[2, M]$. 
Proof. The argument is by induction on $M$. For $M=2$ the claim is obvious. Consider $M \geq 3$. Set $K_{m}=\sum_{i=2}^{m} c_{i}$. It suffices to show the claimed bound for $K_{M}$ (for the others the claim is clear by hypothesis). Note that $M K_{M}=\sum_{m=2}^{M-1} K_{m}+\sum_{i=2}^{M} i c_{i}$. Thus,

$$
M K_{M} \leq C \sum_{m=2}^{M-1}\left(1+\sum_{i=3}^{m} 1 / i\right)+C M=C M\left(1+\sum_{i=3}^{M} 1 / i\right),
$$

where the last equality can be seen, for example, by another inductive argument.

Lemma 5.7. Let $n=p \in \mathbb{P}$.

1. For every $t \in[1, p-1]$, we have $m(G, t+1) \leq\left\lfloor\frac{(p-1)^{2}}{\lfloor p / t\rfloor}\right\rfloor+1$.

2. If $S$ is a zero-sum sequence over $G^{\bullet}$, then $\min \mathrm{L}(S) \leq \min \left\{\frac{|S|}{2}, \frac{2|S|}{p+5}+2(p-1)\left(\frac{1}{2}+\log \left(\frac{p+3}{2}\right)\right)\right\}$.

Proof. 1. This follows from Lemma 5.5 .

2. Clearly, we have $\min \mathrm{L}(S) \leq \max \mathrm{L}(S) \leq \frac{|S|}{2}$. Thus it suffices to prove that $\min \mathrm{L}(S)$ is bounded above by the second term in the above set. To do so, we show that there exists a factorization $S=$ $U_{1} \cdot \ldots \cdot U_{t}$, where $U_{1}, \ldots, U_{t} \in \mathcal{A}(G)$ and $t$ is bounded above by the given term. We construct $U_{1}, \ldots, U_{t}$ recursively. Indeed, for $i \in[1, t]$, let $U_{i}$ be a minimal zero-sum subsequence of $S\left(\prod_{j=1}^{i-1} U_{j}\right)^{-1}$, whose length is maximal possible. Now we use 1 . to obtain an upper bound on $t$. For every $k \in[2, p]$, let $n_{k}$ be the number of $U_{i}$ such that $\left|U_{i}\right|=k$. For every $m \in[2, p-1]$, the construction of $U_{i}$ and 1 . imply that

$$
\sum_{i=2}^{m} i n_{i} \leq\left\lfloor\frac{(p-1)^{2}}{\lfloor p / m\rfloor}\right\rfloor
$$

If $m \leq \frac{p+3}{2}$, then $\lfloor p / m\rfloor \geq \frac{p-m+1}{m} \geq \frac{p-1}{2 m}$. Therefore, from (5.2) we infer that

$$
\sum_{i=2}^{m} i n_{i} \leq 2(p-1) m
$$

holds for every $m \in\left[2, \frac{p+3}{2}\right]$.

By equation (5.3) and Lemma 5.6 we obtain that

$$
\sum_{i=2}^{m} n_{i} \leq 2(p-1)\left(1+\frac{1}{3}+\frac{1}{4}+\ldots+\frac{1}{m}\right)
$$

holds for every $m \in\left[2, \frac{p+3}{2}\right]$. Especially,

$$
\sum_{i=2}^{\frac{p+3}{2}} n_{i} \leq 2(p-1)\left(\frac{1}{2}+\sum_{i=2}^{\frac{p+3}{2}} \frac{1}{i}\right) \leq 2(p-1)\left(\frac{1}{2}+\log \left(\frac{p+3}{2}\right)\right) .
$$

Since $\sum_{i \geq \frac{p+5}{2}} i n_{i} \leq|S|$, we have $\sum_{i \geq \frac{p+5}{2}} n_{i} \leq \frac{2|S|}{p+5}$. Hence,

$$
t=\sum_{i=2}^{p} n_{i} \leq \frac{2|S|}{p+5}+2(p-1)\left(\frac{1}{2}+\log \left(\frac{p+3}{2}\right)\right) .
$$

Lemma 5.8. Let $n=p^{\alpha}$, where $p \in \mathbb{P}$ and $\alpha \geq 2$, and let $S$ be a zero-sum sequence over $G$ such that $\operatorname{ord}(g)=n$ for all $g \in \operatorname{supp}(S)$. Then $\min \mathrm{L}(S) \leq \min \left\{\frac{|S|}{2}, \frac{2|S|}{n+1}+3(n-1)\left(\frac{1}{2}+\log \left(\frac{n}{2}\right)\right)\right\}$. 
Proof. As in Lemma 5.7,2., it suffices to show that there exists a factorization $S=U_{1} \cdot \ldots \cdot U_{t}$, where $U_{1}, \ldots, U_{t} \in \mathcal{A}(G)$ and $t$ is bounded above by the second term in the above set. We construct $U_{1}, \ldots, U_{t}$ recursively. Indeed, for $i \in[1, t]$, let $U_{i}$ be a minimal zero-sum subsequence of $S\left(\prod_{j=1}^{i-1} U_{j}\right)^{-1}$, whose length is maximal possible. We are going to use Lemma 5.5 to get an upper bound on $t$. For every $k \in[2, n]$, let $n_{k}$ be the number of $U_{i}$ such that $\left|U_{i}\right|=k$. For every $m \in[3, n-1]$, the construction of $U_{i}$ and Lemma 5.5 imply that

$$
\sum_{i=2}^{m-1} i n_{i} \leq \frac{\phi(n)(n-1)}{\phi_{\left\lfloor\frac{n}{m}\right\rfloor}(n)}
$$

If $m \leq \frac{n+2}{2}$ then $\phi_{\left\lfloor\frac{n}{m}\right\rfloor}(n)=\left\lfloor\frac{n}{m}\right\rfloor-\left\lfloor\frac{\left\lfloor\frac{n}{m}\right\rfloor}{p}\right\rfloor \geq\left\lfloor\frac{n}{m}\right\rfloor\left(1-\frac{1}{p}\right) \geq \frac{n-m+1}{m}\left(1-\frac{1}{p}\right) \geq \frac{n}{2 m}\left(1-\frac{1}{p}\right)=\frac{\phi(n)}{2 m}$. It follows from (5.4) that

$$
\sum_{i=2}^{m-1} i n_{i} \leq 2 m(n-1) \leq 3(m-1)(n-1) .
$$

Therefore, for every $m \in\left[2, \frac{n}{2}\right]$, we have

$$
\sum_{i=2}^{m} i n_{i} \leq 3(n-1) m
$$

It follows from (5.5), applying Lemma 5.6, that

$$
\sum_{i=2}^{m} n_{i} \leq 3(n-1)\left(1+\frac{1}{3}+\frac{1}{4}+\ldots+\frac{1}{m}\right)
$$

holds for every $m \in\left[2, \frac{n}{2}\right]$. Especially,

$$
\sum_{i=2}^{\frac{n}{2}} n_{i} \leq 3(n-1)\left(\frac{1}{2}+\sum_{i=2}^{\frac{n}{2}} \frac{1}{i}\right) \leq 3(n-1)\left(\frac{1}{2}+\log \left(\frac{n}{2}\right)\right) .
$$

Since $\sum_{i>\frac{n}{2}} i n_{i}=\sum_{i \geq \frac{n+1}{2}} i n_{i} \leq|S|, \sum_{i>\frac{n}{2}} n_{i} \leq \frac{|S|}{\frac{n+1}{2}}$. Hence,

$$
t=\sum_{i=2}^{n} n_{i} \leq \frac{2|S|}{n+1}+3(n-1)\left(\frac{1}{2}+\log \left(\frac{n}{2}\right)\right) \text {. }
$$

Lemma 5.9. Let $n=p^{\alpha}$, where $p \in \mathbb{P}$ and $\alpha \geq 2$, and let $S$ be a zero-sum sequence over $G^{\bullet}$. For every positive divisor $d>1$ of $n$, let $N_{d}$ denote the number of the terms of $S$ which have order $d$. Then $\min \mathrm{L}(S) \leq \min \left\{\frac{|S|}{2},-2 \alpha+\frac{2 p^{\alpha+1}}{p-1}+\sum_{i=1}^{\alpha} \frac{2 N_{p^{i}}}{p^{i}+1}+3 \sum_{i=1}^{\alpha}\left(p^{i}-1\right) \log \frac{p^{i}}{2}\right\}$.

Proof. Clearly, it suffices to prove that $\min \mathrm{L}(S)$ is bounded above by the second term in the above set. For every $i \in[1, \alpha]$, let $S_{i}$ denote the subsequence of $S$ consisting of all terms with order $p^{i}$, let $T_{i}$ be a zero-sum subsequence of $S_{i}$ with maximal possible length, and set $T_{i}^{\prime}=S_{i} T_{i}^{-1}$. Therefore

$$
S=S_{1} \cdot \ldots \cdot S_{\alpha}=\prod_{i=1}^{\alpha} T_{i}^{\prime} \prod_{i=1}^{\alpha} T_{i} \text { and } \prod_{i=1}^{\alpha} T_{i}^{\prime} \text { has sum zero. }
$$

By the maximality of $T_{i}$ we infer that $\left|T_{i}^{\prime}\right| \leq p^{i}-1$ for every $i \in[1, \alpha]$. Hence,

$$
\left|\prod_{i=1}^{\alpha} T_{i}^{\prime}\right| \leq \sum_{i=1}^{\alpha}\left(p^{i}-1\right) \leq p^{\alpha} \frac{p}{p-1}-\alpha .
$$


Therefore,

$$
\min \mathrm{L}(S) \leq \min \mathrm{L}\left(\prod_{i=1}^{\alpha} T_{i}^{\prime}\right)+\sum_{i=1}^{\alpha} \min \mathrm{L}\left(T_{i}\right) \leq \frac{\left|\prod_{i=1}^{\alpha} T_{i}^{\prime}\right|}{2}+\sum_{i=1}^{\alpha} \min \mathrm{L}\left(T_{i}\right) .
$$

It follows from (5.6) that

$$
\min \mathrm{L}(S) \leq \frac{p^{\alpha} \frac{p}{p-1}-\alpha}{2}+\sum_{i=1}^{\alpha} \min \mathrm{L}\left(T_{i}\right) .
$$

By Lemma 5.7,2 and Lemma 5.8 we obtain that

$$
\min \mathrm{L}\left(T_{i}\right) \leq \frac{2\left|T_{i}\right|}{p^{i}+1}+3\left(p^{i}-1\right)\left(\frac{1}{2}+\log \frac{p^{i}}{2}\right)
$$

holds for every $i \in[1, \alpha]$. It follows from (5.6) that

$$
\begin{aligned}
\min \mathrm{L}(S) & \leq \frac{p^{\alpha} \frac{p}{p-1}-\alpha}{2}+\sum_{i=1}^{\alpha}\left(\frac{2\left|T_{i}\right|}{p^{i}+1}+3\left(p^{i}-1\right)\left(\frac{1}{2}+\log \frac{p^{i}}{2}\right)\right) \\
& =\frac{p^{\alpha+1}-\alpha(p-1)}{2(p-1)}+\sum_{i=1}^{\alpha} \frac{2\left|T_{i}\right|}{p^{i}+1}+\frac{3}{2} \sum_{i=1}^{\alpha}\left(p^{i}-1\right)+3 \sum_{i=1}^{\alpha}\left(p^{i}-1\right) \log \frac{p^{i}}{2} \\
& =2 \frac{p^{\alpha+1}-\alpha(p-1)}{p-1}+\sum_{i=1}^{\alpha} \frac{2\left|T_{i}\right|}{p^{i}+1}+3 \sum_{i=1}^{\alpha}\left(p^{i}-1\right) \log \frac{p^{i}}{2} \\
& \leq 2 \frac{p^{\alpha+1}-\alpha(p-1)}{p-1}+\sum_{i=1}^{\alpha} \frac{2 N_{p^{i}}}{p^{i}+1}+3 \sum_{i=1}^{\alpha}\left(p^{i}-1\right) \log \frac{p^{i}}{2} \\
& =-2 \alpha+\frac{2 p^{\alpha+1}}{p-1}+\sum_{i=1}^{\alpha} \frac{2 N_{p^{i}}}{p^{i}+1}+3 \sum_{i=1}^{\alpha}\left(p^{i}-1\right) \log \frac{p^{i}}{2} .
\end{aligned}
$$

We need certain bounds for Legendre's totient function. We establish what we need in the two subsequent lemmas in a self-contained way.

Lemma 5.10. Let $n=p_{1}^{\alpha_{1}} \cdot \ldots \cdot p_{s}^{\alpha_{s}}$, where $s \geq 2, \alpha_{1}, \ldots, \alpha_{s} \in \mathbb{N}$, and $p_{1}, \ldots, p_{s} \in \mathbb{P}$ are distinct. If $m \geq 2^{s+1} \sqrt{2 s-1}$ then $\phi_{m}(n) \geq \frac{m}{2} \prod_{i=1}^{s}\left(1-\frac{1}{p_{i}}\right)=\frac{m \phi(n)}{2 n}$.

Proof. By the inclusion-exclusion principle we know that

$$
\begin{aligned}
\phi_{m}(n) & =m-\sum_{i=1}\left\lfloor\frac{m}{p_{i}}\right\rfloor+\sum_{1 \leq i<j \leq s}\left\lfloor\frac{m}{p_{i} p_{j}}\right\rfloor-\ldots+(-1)^{s}\left\lfloor\frac{m}{p_{1} \ldots \ldots \cdot p_{s}}\right\rfloor \\
& \geq m-\sum_{i=1} \frac{m}{p_{i}}+\sum_{1 \leq i<j \leq s}\left(\frac{m}{p_{i} p_{j}}-1\right)-\sum_{1 \leq i<j<k} \frac{m}{p_{i} p_{j} p_{k}}+\sum_{1<i<j<k<l}\left(\frac{m}{p_{i} p_{j} p_{k} p_{l}}-1\right)-\ldots \\
& =m \prod_{i=1}^{s}\left(1-\frac{1}{p_{i}}\right)-\left(\left(\begin{array}{c}
s \\
2
\end{array}\right)+\left(\begin{array}{c}
s \\
4
\end{array}\right)+\ldots\right)=m \prod_{i=1}^{s}\left(1-\frac{1}{p_{i}}\right)-2^{s-1} .
\end{aligned}
$$

Therefore,

$$
\phi_{m}(n) \geq m \prod_{i=1}^{s}\left(1-\frac{1}{p_{i}}\right)-2^{s-1} .
$$

It is easy to see that $p_{i} \geq 2 i-1$ for all $i \in[2, s]$. Therefore,

$$
\prod_{i=1}^{s}\left(1-\frac{1}{p_{i}}\right) \geq \frac{1}{2} \prod_{i=2}^{s} \frac{2 i-2}{2 i-1} \text {. }
$$

Since $\frac{2 i-2}{2 i-1} \geq \frac{2 i-3}{2 i-2}$ holds for every $i \in[2, s]$, we obtain that

$$
\left(\prod_{i=2}^{s} \frac{2 i-2}{2 i-1}\right)^{2} \geq \prod_{i=2}^{s} \frac{2 i-2}{2 i-1} \prod_{i=2}^{s} \frac{2 i-3}{2 i-2}=\prod_{i=1}^{2 s-2} \frac{i}{i+1}=\frac{1}{2 s-1} .
$$

It follows that

$$
\prod_{i=2}^{s} \frac{2 i-2}{2 i-1} \geq \frac{1}{\sqrt{2 s-1}}, \quad \text { and hence by (5.9) } \quad \prod_{i=1}^{s}\left(1-\frac{1}{p_{i}}\right) \geq \frac{1}{2 \sqrt{2 s-1}} .
$$

Since $m \geq 2^{s+1} \sqrt{2 s-1}$, from (5.10) we deduce that $\frac{m}{2} \prod_{i=1}^{s}\left(1-\frac{1}{p_{i}}\right) \geq 2^{s-1}$. It follows from (5.8) that $\phi_{m}(n) \geq m \prod_{i=1}^{s}\left(1-\frac{1}{p_{i}}\right)-2^{s-1} \geq \frac{m}{2} \prod_{i=1}^{s}\left(1-\frac{1}{p_{i}}\right)$. 
Lemma 5.11. Let $n=p_{1}^{\alpha_{1}} \cdot \ldots \cdot p_{s}^{\alpha_{s}}$, where $s \geq 2, \alpha_{1}, \ldots, \alpha_{s} \in \mathbb{N}$, and $p_{1}, \ldots, p_{s} \in \mathbb{P}$ are distinct. If $t \leq \frac{n+1}{2^{s+1} \sqrt{2 s-1}+1}$ then $\phi_{\left\lfloor\frac{n}{t}\right\rfloor}(n) \geq \frac{n}{2.2 t} \prod_{i=1}^{s}\left(1-\frac{1}{p_{i}}\right)=\frac{\phi(n)}{2.2 t}$.

Proof. From $t \leq \frac{n+1}{2^{s+1} \sqrt{2 s-1}+1}$ we obtain that $\left\lfloor\frac{n}{t}\right\rfloor \geq \frac{n-t+1}{t} \geq 2^{s+1} \sqrt{2 s-1}$. Lemma 5.10 implies that

$$
\phi_{\left\lfloor\frac{n}{t}\right\rfloor}(n) \geq \frac{1}{2}\left\lfloor\frac{n}{t}\right\rfloor \frac{\phi(n)}{n} \geq \frac{n-t+1}{2 t} \frac{\phi(n)}{n} .
$$

Since $s \geq 2$, we infer that $t \leq \frac{n+1}{2^{s+1} \sqrt{2 s-1}+1} \leq \frac{n+1}{8 \sqrt{3}+1}<\frac{n+11}{11}$. Therefore, $\frac{n-t+1}{2 t} \geq \frac{n}{2.2 t}$ and the result follows from (5.11).

Lemma 5.12. Let $n=p_{1}^{\alpha_{1}} \cdot \ldots \cdot p_{s}^{\alpha_{s}}$, where $s \geq 2, \alpha_{1}, \ldots, \alpha_{s} \in \mathbb{N}$, and $p_{1}, \ldots, p_{s} \in \mathbb{P}$ are distinct, and let $S$ be a zero-sum sequence over $G$ such that $\operatorname{ord}(g)=n$ for all $g \in \operatorname{supp}(S)$. Then $\min \mathrm{L}(S) \leq$ $\min \left\{\frac{|S|}{2}, \frac{|S|}{\left[\frac{n+1}{2^{s+1} \sqrt{2 s-1+1}}\right\rfloor}+3.3(n-1)\left(\frac{1}{2}+\log \left(\left\lfloor\frac{n+1}{2^{s+1} \sqrt{2 s-1}+1}-1\right\rfloor\right)\right)\right\}$.

Proof. For ease of notation, set $u=\frac{n+1}{2^{s+1} \sqrt{2 s-1}+1}$. It suffices to show that there is a factorization $S=$ $U_{1} \cdot \ldots \cdot U_{t}$, where $U_{1}, \ldots, U_{t} \in \mathcal{A}(G)$ and $t$ is bounded above by the second term in the above set, and again we construct $U_{1}, \ldots, U_{t}$ recursively. For $i \in[1, t]$, let $U_{i}$ be a minimal zero-sum subsequence of $S\left(\prod_{j=1}^{i-1} U_{j}\right)^{-1}$ with maximal possible length. We use Lemma 5.5 and Lemma 5.11 to study $t$. For every $k \in[2, n]$, let $n_{k}$ be the number of $U_{i}$ such that $\left|U_{i}\right|=k$. For every $m \in[3, n-1]$, the construction of $U_{i}$ and Lemma 5.5 imply that

$$
\sum_{i=2}^{m-1} i n_{i} \leq \frac{\phi(n)(n-1)}{\phi_{\left\lfloor\frac{n}{m}\right\rfloor}(n)}
$$

If $m \leq u$, then by Lemma 5.11 we have $\phi_{\left\lfloor\frac{n}{m}\right\rfloor}(n) \geq \frac{\phi(n)}{2.2 m}$. It follows from (5.12) that

$$
\sum_{i=2}^{m-1} i n_{i} \leq 2.2 m(n-1) \leq 3.3(m-1)(n-1) \text {. }
$$

Therefore, for every $m \in[2, u-1]$, we have

$$
\sum_{i=2}^{m} i n_{i} \leq 3.3(n-1) m
$$

Applying Lemma 5.6 we deduce that

$$
\sum_{i=2}^{m} n_{i} \leq 3.3\left(1+\frac{1}{3}+\frac{1}{4}+\ldots+\frac{1}{m}\right)(n-1)
$$

holds for every $m \in[2, u-1]$. Especially,

$$
\sum_{i=2}^{\lfloor u-1\rfloor} n_{i} \leq 3.3(n-1)\left(\frac{1}{2}+\sum_{i=2}^{\lfloor u-1\rfloor} \frac{1}{i}\right) \leq 3.3(n-1)\left(\frac{1}{2}+\log (\lfloor u-1\rfloor)\right) .
$$

Since $\sum_{i \geq\lfloor u\rfloor} i n_{i} \leq|S|, \sum_{i \geq\lfloor u\rfloor} n_{i} \leq \frac{\lfloor S \mid}{\lfloor u\rfloor}$. Hence,

$$
t=\sum_{i=2}^{n} n_{i} \leq \frac{|S|}{\lfloor u\rfloor}+3.3(n-1)\left(\frac{1}{2}+\log (\lfloor u-1\rfloor)\right) .
$$

Lemma 5.13. Let $n=p_{1}^{\alpha_{1}} \cdot \ldots \cdot p_{s}^{\alpha_{s}}$, where $s \geq 2, \alpha_{1}, \ldots, \alpha_{s} \in \mathbb{N}$, and $p_{1}, \ldots, p_{s} \in \mathbb{P}$ are distinct. If $n \geq 4376$, then $\left\lfloor\frac{n+1}{2^{s+1} \sqrt{2 s-1}+1}\right\rfloor \geq \frac{n}{2^{s+1} \sqrt{2 s}}$. 
Proof. Clearly, it suffices to prove that

$$
\frac{n+1}{2^{s+1} \sqrt{2 s-1}+1} \geq \frac{n}{2^{s+1} \sqrt{2 s}}+1
$$

which will follow from

$$
\left(\frac{2^{s+1}}{\sqrt{2 s}+\sqrt{2 s-1}}-1\right) n \geq 2^{2 s+2} \sqrt{2 s(2 s-1)} .
$$

By a straightforward computation we get that (5.14) holds for $(s=2$ and $n \geq 250)$, for $(s=3$ and $n \geq 656)$, for $(s=4$ and $n \geq 1707)$, and for $(s=5$ and $n \geq 4376)$. If $s=6$, then $n \geq 2 \times 3 \times 5 \times 7 \times 11 \times 13=$ 30030. Again by a straightforward computation we get that (5.14) holds. Now we proceed by induction. Assume that (5.14) holds for some $s \geq 6$. Then it holds for $s+1$ because

$$
\begin{aligned}
\left(\frac{2^{s+2}}{\sqrt{2(s+1)}+\sqrt{2 s+1}}-1\right) n & \geq\left(\frac{2^{s+1}}{\sqrt{2 s}+\sqrt{2 s-1}}-1\right) \frac{n}{p_{s+1}^{\alpha_{s+1}}} p_{s+1}^{\alpha_{s+1}} \\
& \geq\left(2^{2 s+2} \sqrt{2 s(2 s-1)}\right) p_{s+1}^{\alpha_{s+1}} \\
& \geq\left(2^{2 s+2} \sqrt{2 s(2 s-1)}\right) \times 17 \\
& \geq 2^{2 s+4} \sqrt{2(s+1)(2 s+1)} .
\end{aligned}
$$

Lemma 5.14. Let $n=p_{1}^{\alpha_{1}} \cdot \ldots \cdot p_{r}^{\alpha_{r}}$, where $r \geq 2, \alpha_{1}, \ldots, \alpha_{r} \in \mathbb{N}$, and $p_{1}, \ldots, p_{r} \in \mathbb{P}$ are distinct, and let $S$ be a zero-sum sequence over $G^{\bullet}$. For every divisor $d>1$ of $n$, let $N_{d}$ denote the number of the terms of $S$ which have order $d$. Then $\min \mathrm{L}(S) \leq \min \left\{\frac{|S|}{2}, \frac{4.3}{2} \sum_{1<d \mid n}(d-1)+\sum_{1<d \mid n, d \leq 4375} \frac{N_{d}}{2}+\right.$ $\left.\sum_{4376 \leq d \mid n} \frac{2^{\omega(d)+1} \sqrt{2 \omega(d)} N_{d}}{d}+3.3 \sum_{4376 \leq d \mid n}(d-1) \log \left(\left\lfloor\frac{d+1}{2^{\omega(d)+1} \sqrt{2 \omega(d)-1}+1}-1\right\rfloor\right)\right\}$.

Proof. It suffices to show that $\min \mathrm{L}(S)$ is bounded above by the second term in the above set. For every $1<d \mid n$, let $S_{d}$ denote the subsequence of $S$ consisting of all terms with order $d$, let $T_{d}$ be a zero-sum subsequence of $S_{d}$ with maximal possible length, and set $T_{d}^{\prime}=S_{d} T_{d}^{-1}$. Therefore

$$
S=\prod_{1<d \mid n} T_{d}^{\prime} \prod_{1<d \mid n} T_{d} \text { and } \prod_{1<d \mid n} T_{d}^{\prime} \quad \text { has sum zero. }
$$

By the maximality of $T_{d}$ we infer that $\left|T_{d}^{\prime}\right| \leq d-1$ for every $1<d \mid n$, and hence,

$$
\left|\prod_{1<d \mid n} T_{d}^{\prime}\right| \leq \sum_{1<d \mid n}(d-1)
$$

Therefore,

$$
\min \mathrm{L}(S) \leq \min \mathrm{L}\left(\prod_{1<d \mid n} T_{d}^{\prime}\right)+\sum_{1<d \mid n} \min \mathrm{L}\left(T_{d}\right) \leq \frac{\left|\prod_{1<d \mid n} T_{d}^{\prime}\right|}{2}+\sum_{1<d \mid n} \min \mathrm{L}\left(T_{d}\right) .
$$

It follows from (5.15) that

$$
\min \mathrm{L}(S) \leq \frac{\sum_{1<d \mid n}(d-1)}{2}+\sum_{1<d \mid n} \min \mathrm{L}\left(T_{d}\right) .
$$

If $\omega(d) \geq 2$ then by Lemma 5.12 and Lemma 5.13 we obtain that

$$
\min L\left(T_{d}\right) \leq \frac{2^{\omega(d)+1} \sqrt{2 \omega(d)} N_{d}}{d}+3.3(d-1)\left(\frac{1}{2}+\log \left(\left\lfloor\frac{d+1}{2^{\omega(d)+1} \sqrt{2 \omega(d)-1}+1}-1\right\rfloor\right)\right)
$$


holds for every $4376 \leq d \mid n$. By Lemma 5.72 and Lemma 5.8 we obtain that (5.17) is true for all $1<d \mid n$ with $\omega(d)=1$. It follows from (5.16) that

$$
\begin{aligned}
& \min \mathrm{L}(S) \leq \frac{\sum_{1<d \mid n} d-1}{2}+\sum_{1<d \mid n} \min \mathrm{L}\left(T_{d}\right) \\
& \leq \frac{\sum_{1<d \mid n} d-1}{2}+\sum_{1<d \mid n, d \leq 4375} \frac{N_{d}}{2}+\sum_{4376 \leq d \mid n}\left(\frac{2^{\omega(d)+1} \sqrt{2 \omega(d)} N_{d}}{d}+3.3(d-1)\left(\frac{1}{2}+\log \left(\left\lfloor\frac{d+1}{2^{\omega(d)+1} \sqrt{2 \omega(d)-1}+1}-1\right\rfloor\right)\right)\right. \\
& \leq \frac{4.3}{2} \sum_{1<d \mid n}(d-1)+\sum_{1<d \mid n, d \leq 4375} \frac{N_{d}}{2}+\sum_{4376 \leq d \mid n} \frac{2^{\omega(d)+1} \sqrt{2 \omega(d)} N_{d}}{d} \\
& +3.3 \sum_{4376 \leq d \mid n}(d-1) \log \left(\left\lfloor\frac{d+1}{2^{\omega(d)+1} \sqrt{2 \omega(d)-1}+1}-1\right\rfloor\right) .
\end{aligned}
$$

Proof of Theorem 5.2. We may suppose that $H$ is reduced and that $H \hookrightarrow \mathcal{F}(P)$ is a divisor theory with class group $G$. Let $u, v_{1}, \ldots, v_{m}, u_{2}, \ldots, u_{\ell} \in \mathcal{A}(H)$ be such that $u \mid v_{1} \cdot \ldots \cdot v_{m}$, but $u$ divides no proper subproduct, that $v_{1} \cdot \ldots \cdot v_{m}=u u_{2} \cdot \ldots \cdot u_{\ell}$, and that $\max \{\ell, m\}=\mathrm{t}(H, u)=\mathrm{t}(H)$. By Lemma 4.1.2, we have $\mathrm{t}(H) \geq \mathrm{t}(G)>n \geq|u| \geq m$, and hence we get $1+\min \mathrm{L}(w)=\ell=\mathrm{t}(H)>n$, where $w=u^{-1} v_{1} \cdot \ldots \cdot v_{m}$. For $i \in[1, m]$, we set $v_{i}=s_{i} a_{i}$ with $a_{i}, s_{i} \in \mathcal{F}(P) \backslash\{1\}$ and $A_{i}=\boldsymbol{\beta}\left(a_{i}\right)$. We set $S=A_{1} \cdot \ldots \cdot A_{m}$, and observe that $S=\boldsymbol{\beta}(w)$ and that $|S| \leq m(n-1) \leq n(n-1)$. We have to show that $1+\min \mathrm{L}(S)$ is bounded above by the terms given in the statement of the theorem.

CASE 1: $n=p \in \mathbb{P}$.

This follows from Lemma [5.72.

CASE 2: $\quad n=p^{\alpha}$, where $p \in \mathbb{P}$ and $\alpha \geq 2$.

For every divisor $d>1$ of $n$, let $N_{d}$ denote the number of the terms of $S$ which has order $d$. Since $A_{i}$ is zero-sum free, we infer that $A_{i}$ has at most $d-1$ terms which have order $d$, and hence $N_{d} \leq m(d-1) \leq$ $n(d-1)$. Thus it follows from Lemma 5.9 that

$$
\begin{aligned}
\min \mathrm{L}(S) & \leq-2 \alpha+\frac{2 p^{\alpha+1}}{p-1}+\sum_{i=1}^{\alpha} \frac{2 N_{p^{i}}}{p^{i}+1}+3 \sum_{i=1}^{\alpha}\left(p^{i}-1\right) \log \frac{p^{i}}{2} \\
& \leq-2 \alpha+\frac{2 p^{\alpha+1}}{p-1}+\sum_{i=1}^{\alpha} \frac{2 n\left(p^{i}-1\right)}{p^{i}+1}+3 \sum_{i=1}^{\alpha}\left(p^{i}-1\right) \log \frac{p^{i}}{2} \\
& \leq-2 \alpha+\frac{2 p^{\alpha+1}}{p-1}+2 \alpha n+3 \sum_{i=1}^{\alpha}\left(p^{i}-1\right) \log \frac{p^{i}}{2} .
\end{aligned}
$$

CASE 3: $\quad n=p_{1}^{\alpha_{1}} \cdot \ldots \cdot p_{r}^{\alpha_{r}}$, where $r \geq 2, \alpha_{1}, \ldots, \alpha_{r} \in \mathbb{N}$, and $p_{1}, \ldots, p_{r} \in \mathbb{P}$ are distinct.

For every divisor $d>1$ of $n$, let $N_{d}$ denote the number of the terms of $S$ which have order $d$. Since $A_{i}$ is zero-sum free, we infer that $A_{i}$ has at most $d-1$ terms whose order divide $d$. Therefore, $N_{d} \leq m(d-1) \leq n(d-1)$ for each $1<d \mid n$. Now the result follows from Lemma 5.14

Acknowledgement. We thank the referees for reading the paper carefully. They have provided a list of helpful comments which, among others, led to a more general version of Lemma 4.3.

\section{REFERENCES}

[1] N.R. Baeth and R.A. Wiegand, Factorization theory and decomposition of modules, Am. Math. Mon. 120 (2013), 3 34 .

[2] V. Blanco, P. A. García-Sánchez, and A. Geroldinger, Semigroup-theoretical characterizations of arithmetical invariants with applications to numerical monoids and Krull monoids, Illinois J. Math. 56 (2012).

[3] Gyu-Whan Chang, Every divisor class of Krull monoid domains contains a prime ideal, J. Algebra 336 (2011), 370 377.

[4] S.T. Chapman, P.A. García-Sánchez, and D. Llena, The catenary and tame degree of numerical monoids, Forum Math. 21 (2009), $117-129$.

[5] S.T. Chapman, P.A. García-Sánchez, D. Llena, V. Ponomarenko, and J.C. Rosales, The catenary and tame degree in finitely generated commutative cancellative monoids, Manuscr. Math. 120 (2006), 253 - 264.

[6] A. Facchini, Krull monoids and their application in module theory, Algebras, Rings and their Representations (A. Facchini, K. Fuller, C. M. Ringel, and C. Santa-Clara, eds.), World Scientific, 2006, pp. $53-71$.

[7] A. Facchini, W. Hassler, L. Klingler, and R. Wiegand, Direct-sum decompositions over one-dimensional CohenMacaulay local rings, Multiplicative Ideal Theory in Commutative Algebra (J.W. Brewer, S. Glaz, W. Heinzer, and B. Olberding, eds.), Springer, 2006, pp. $153-168$. 
[8] M. Fontana, E. Houston, and T. Lucas, Factoring Ideals in Integral Domains, Lecture Notes of the Unione Matematica Italiana, vol. 14, Springer, 2013.

[9] A. Geroldinger, Additive group theory and non-unique factorizations, Combinatorial Number Theory and Additive Group Theory (A. Geroldinger and I. Ruzsa, eds.), Advanced Courses in Mathematics CRM Barcelona, Birkhäuser, 2009 , pp. $1-86$.

[10] A. Geroldinger and D.J. Grynkiewicz, On the arithmetic of Krull monoids with finite Davenport constant, J. Algebra 321 (2009), $1256-1284$.

[11] A. Geroldinger, D.J. Grynkiewicz, G.J. Schaeffer, and W.A. Schmid, On the arithmetic of Krull monoids with infinite cyclic class group, J. Pure Appl. Algebra 214 (2010), 2219 - 2250.

[12] A. Geroldinger and F. Halter-Koch, Non-Unique Factorizations. Algebraic, Combinatorial and Analytic Theory, Pure and Applied Mathematics, vol. 278, Chapman \& Hall/CRC, 2006.

[13] A. Geroldinger and W. Hassler, Arithmetic of Mori domains and monoids, J. Algebra 319 (2008), 3419 - 3463.

[14] Local tameness of $v$-noetherian monoids, J. Pure Appl. Algebra 212 (2008), 1509 - 1524.

[15] A. Geroldinger and F. Kainrath, On the arithmetic of tame monoids with applications to Krull monoids and Mori domains, J. Pure Appl. Algebra 214 (2010), 2199 - 2218.

[16] A. Geroldinger, M. Liebmann, and A. Philipp, On the Davenport constant and on the structure of extremal sequences, Period. Math. Hung. 64 (2012), $213-225$.

[17] F. Halter-Koch, Ideal Systems. An Introduction to Multiplicative Ideal Theory, Marcel Dekker, 1998.

[18] F. Kainrath, Arithmetic of Mori domains and monoids: the Global Case, submitted.

[19] _ The distribution of prime divisors in finitely generated domains, Manuscr. Math. 100 (1999), 203 - 212.

[20] H. Kim and Y. S. Park, Krull domains of generalized power series, J. Algebra 237 (2001), $292-301$.

[21] M. Omidali, The catenary and tame degree of numerical monoids generated by generalized arithmetic sequences, Forum Math. 24 (2012), $627-640$.

[22] A. Philipp, A characterization of arithmetical invariants by the monoid of relations II: The monotone catenary degree and applications to semigroup rings, Semigroup Forum, to appear.

[23] - A characterization of arithmetical invariants by the monoid of relations, Semigroup Forum $\mathbf{8 1}$ (2010), 424 434 .

[24], A precise result on the arithmetic of non-principal orders in algebraic number fields, J. Algebra Appl. 11 (2012).

[25] A. Reinhart, On integral domains that are C-monoids, Houston J. Math., to appear.

Center for Combinatorics, Nankai University, Tianjin 300071, P.R. China

E-mail address: wdgao_1963@aliyun.com

Institut für Mathematik und Wissenschaftliches Rechnen, Karl-Franzens-Universität Graz, HeinrichStrasse 36, 8010 Graz, Austria

E-mail address: alfred.geroldinger@uni-graz.at

Université Paris 13, Sorbonne Paris Cité, LAGA, CNRS, UMR 7539, Université Paris 8, F-93430, Villetaneuse, FRANCE

E-mail address: schmid@math.univ-paris13.fr 